\title{
Chirality change in string theory
}

\section{Michael R. Douglas}

Department of Physics and Astronomy, Rutgers University

Piscataway, NJ 08855-0849, U.S.A., and

I.H.E.S., Le Bois-Marie, Bures-sur-Yvette, 91440 France, and

Caltech 452-48, Pasadena, CA 91125, U.S.A.

E-mail: mrd@physics.rutgers.edu

\section{Chengang Zhou}

Department of Astronomy and Physics, University of Kentucky Lexington, $K Y$ 40506, U.S.A.

E-mail: czhou@pa.uky.edu

ABSTRACT: It is known that string theory compactifications leading to low energy effective theories with different chiral matter content (e.g. different numbers of standard model generations) are connected through phase transitions, described by non-trivial quantum fixed point theories.

We point out that such compactifications are also connected on a purely classical level, through transitions that can be described using standard effective field theory. We illustrate this with examples, including some in which the transition proceeds entirely through supersymmetric configurations.

KEYwords: Superstrings and Heterotic Strings, D-branes, Supersymmetric Effective Theories. 


\section{Contents}

1. Introduction 1

2. Toy models 1

2.1 Kaluza-Klein reduction on $S^{1}$

2.2 Connections on $S^{2}$

2.3 Fermion zero modes on $S^{2}$ and change of chirality 11

2.3.1 What is going on 12

2.4 The torus $T^{2}$ and the brane picture 14

2.5 Holomorphic bundles, splitting type, and Yang-Mills connections 16

3. Chirality change on compact Calabi-Yau in heterotic string theory 18

3.1 Chirality from reducible connections 18

3.2 Finiteness of the number of possible $c_{3}$ 's 19

3.3 Explicit search on the quintic 20

4. Smooth chirality change in type-IIA orientifold 23

4.1 Type-IIA orientifold compactification on $T^{6} / \mathbb{Z}_{2} \times \mathbb{Z}_{2}$

4.2 Smooth brane deformation and supersymmetric chirality change 26

5. Conclusions 32

A. Vector bundles $V_{(1,1,1,1,2,2 ; 4,4)}$ on quintic Calabi-Yau 34

B. On finiteness of number of solutions to monad construction of vector bundles

\section{Introduction}

If string theory is truly the right unified theory, it has to make contact with the real world by reproducing the Standard Model at low energies. One of the signatures of the Standard Model is the existence of three generations of leptons and quarks, and one of the early successes of string theory was a natural mechanism producing a multiplicity of generations minus antigenerations, in the context of heterotic string compactification on a Calabi-Yau threefold $\left(\mathrm{CY}_{3}\right) \mathrm{M}$, using simple topological properties of the $\mathrm{CY}_{3}$ [1].

Subsequent work led to many generalizations and other constructions leading to grand unified theories with $\mathcal{N}=1$ supersymmetry at a low scale, not to mention more radical alternatives. In many ways the simplest generalization is to keep as the starting point $d=10, \mathcal{N}=1$ supergravity/super Yang-Mills with $E_{8} \times E_{8}$ or $\mathrm{SO}(32)$ gauge group and 
with the stringy corrections needed for anomaly cancellation, and still compactify on a $\mathrm{CY}_{3}$, only generalizing by taking a general background gauge connection for a vector bundle $V$ satisfying anomaly cancellation constraints [2, 3. These are often referred to as " $(0,2)$ " models from the world-sheet supersymmetry of the heterotic sigma model; in nonperturbative heterotic string theory this class of models is further generalized [ number of generations minus antigenerations, which we will denote $N_{\text {gen }}$, is still determined by the choice of $V$. Most of the other constructions, including type-IIa and IIb orientifold constructions, are dual to these two constructions in a fairly clear way.

This picture raises many questions, such as: for which choices of CY $M$ does there exist a three generation model? How do we find its low energy lagrangian? Which choices lead to stable vacua with low scale $\mathcal{N}=1$ supersymmetry? If there are many, which additional criteria if any pick out the physically relevant one? Somewhat simpler but still relevant questions are, for a given CY $M$, what are possible values of $N_{\text {gen }}$, and are vacua with different $N_{\text {gen }}$ connected in the configuration space of string theory?

In this paper we discuss whether vacua with different chiral matter spectrum, including standard models with different numbers of generations, are connected in string theory. Previous work on this question includes that of Kachru and Silverstein [5 who discuss a mechanism which connects such vacua through a non-trivial phase transition, whose IR behavior is governed by the dimensional reduction of the phase transition associated to the $(1,0)$ superconformal theory in six dimensions. Further work supporting this idea appears in [6].

One motivation for the present work is to refute a (still) common belief that the number of generations provides an "index" which in classical physics is conserved under continuous variations of the background, so that such transitions could only proceed through a phase transition.

This is false and in fact there is a very simple argument that all vacua obtained by making different choices of $V$ must be connected already on the classical level, without going through non-classical phase transitions. We will give this in more detail below, but the essence in the heterotic string construction is just the following. We recall that the low energy gauge group is the commutant in the ten dimensional gauge group (say) $E_{8} \times E_{8}$ of the structure group $G$ of the bundle $V$. To get a realistic grand unified gauge group, we take $V$ to have structure group $\mathrm{SU}(k)$ (for example $k=4$ leads to unbroken $\mathrm{SO}(10)$ ). The number of generations is then $N_{\text {gen }}=c_{3}(V) / 2$, as follows from the index theorem [7].

Now for bundles with structure group $\mathrm{SU}(k), c_{3}(V)$ is a topological invariant. On the other hand, if we allow general motions in configuration space (possibly through a potential barrier), nothing prevents us from deforming the connection on $V$ into the full ten dimensional gauge group. Now, for $E_{8}$ and $\operatorname{Spin}(32) / \mathbb{Z}_{2}$ bundles, $c_{3}(V)$ is not a topological invariant; in fact it cannot even be defined: its definition uses the third order symmetric invariant $d^{a b c}$, which does not exist for these Lie algebras. Indeed, as is familiar from other discussions [20], all $E_{8}$ vector bundles on a manifold $M$ of (real) dimension less than 15 with a specified rank and second Chern class $c_{2}(V)$ are connected by smooth deformations. Such a deformation will correspond to a path in the configuration space of the full string theory, connecting vacua with two possibly different values of $N_{\text {gen }}$. 
Naively, this might be thought paradoxical as chiral matter cannot be lifted by varying uncharged fields or parameters in a low energy effective lagrangian. The obvious loophole which this suggests is that chiral matter must be lifted by giving vevs to charged fields, as is familiar even in the standard model. When the gauge connection in $V$ deforms into $E_{8}$ (or any larger group containing the original $\mathrm{SU}(k)$ ), some or all of the low energy gauge symmetry is broken. If the chiral matter is nonchiral under the remaining unbroken gauge symmetry, there is no barrier to giving it a mass. As an example, after a deformation breaking $\mathrm{SO}(10)$ to $\mathrm{SO}(9)$, a chiral spinor in the 16 of $\mathrm{SO}(10)$ becomes a nonchiral spinor of $\mathrm{SO}(9)$, which can be lifted.

The remaining question is how the chiral matter can remain massive when the original gauge symmetry is restored with different $c_{3}$. The answer to this question is that the final result is a new model in which the original gauge symmetry is not restored. Rather, an isomorphic but different copy of the gauge group becomes unbroken. One should think of the low energy gauge group as a small unbroken subgroup of a large (even infinite dimensional) gauge group, the group of all gauge transformations of $E_{8}$ gauge theory in six dimensions, almost all of which is broken by the background. Even if the unbroken gauge groups $G$ are isomorphic on the two sides of the transition, they can be two different embeddings of this gauge group in the large gauge group.

The minimal effective field theory realization of this phenomenon is in terms of $G \times G$ gauge theory, with matter chosen to always break at least one copy of $G$, and along the interpolating path break to a subgroup of $G$. We will exhibit such effective lagrangians in section 2 .

These simple arguments more or less suffice for a basic physical understanding of the phenomenon. Most of the paper is devoted to seeing how this works in string theory examples, using fairly elementary methods, and dispelling various paradoxes which arise.

We also consider some more detailed questions. One of these is to ask what is the potential barrier to such a transition, a question which might enter in early cosmology or in using some hypothetical "vacuum selection principle." At present there is no good proposal for such a principle, and at present we do not have good control over nonperturbative corrections to the potential, but the question is still interesting to consider.

One expects the mechanism of [5] to involve no potential barrier, because it goes through a superconformal theory, with no preferred energy scale. As for the classical mechanism we discuss, on general grounds, one might expect variations of the gauge connection on a compact manifold of "size" $R$ to go through a potential barrier of height $V \sim 1 / g_{Y M 4}^{2} R^{4}$. This can be made small at large radius or strong coupling but would be non-zero.

Is it possible that examples exist with no potential barrier? To get at this question, we discuss explicit string theory examples. We start in section 2 with toy models, gauge theory compactified on $S^{1}$ and $S^{2}$, which are easy to work out in full detail. The infinite dimensional gauge groups which arise here are the loop groups $\hat{G}$. One already finds on $S^{2}$ and $T^{2}$ that chirality change in a sense is possible.

We then consider a more physical example, gauge theory compactified on $T^{2}$. If one considers saddle points of the potential as vacua (nonsupersymmetric and with tachyons), one can find paths between such vacua which are chirality changing. This theory sits in 
string theory compactified to eight dimensions, so already one can have chirality change in this extremely simple example. It can be made completely transparent by realizing the gauge theory on Dirichlet branes and doing a T-duality: one has a simple picture of brane recombination which changes the intersection number.

In section 1 we discuss aspects of the same problem on the quintic CY. We exhibit many vector bundles with different values of $c_{3}(V)$, which shows that such processes are possible here as well, and discuss the general problem of finding out which values of $N_{\text {gen }}$ can arise. We check, using arguments given in the eppendix, that there indeed exists a stable vector bundle with $c_{3}(V)$ different from that that of the tangent bundle, which can be used in heterotic compactification. Finally, we give an argument (also in the appendix) that the two term monad construction leads to a finite number of vector bundles satisfying the anomaly cancellation condition on a large class of Calabi-Yau manifolds.

In section 5 we discuss a similar brane construction in using D6-branes in orientifolded type-IIa theory, appearing in work of Cvetic, Shiu and Uranga [8] which in fact realizes such a classical chirality changing transition. We will go a bit further and prove that in a very similar construction, this transition in fact proceeds through supersymmetric configurations, and thus there is no potential barrier. We suspect this is fairly general and is true of the construction of [B] as well.

Appendix A contains the conclusions and further directions for study.

\section{Toy models}

We start off by writing down effective lagrangians which illustrate the basic point. In four space-time dimensions, since the two chiralities of spinor are complex conjugate (or, equivalently, since one can write Majorana mass terms), chiral spinors only exist in gauge theory. The discussion is simplest if all spinors are taken to be Weyl of one chirality; then a theory is chiral if the spinors live in a complex representation of the gauge group. The same discussion applies to eight space-time dimensions.

We will write $\mathcal{N}=1$ supersymmetric effective lagrangians, being more relevant to the later discussion. One could expand in components and drop fields to get simpler nonsupersymmetric examples.

One can already have complex representations and chiral spinors in U(1) gauge theories, so these provide the simplest examples. A simple anomaly free chiral spectrum for $\mathrm{U}(1)$ gauge theory is to take one field $\phi^{(q)}$ with charge $q$, and $q^{3}$ fields $\phi_{i}^{(-1)}$ of charge -1 . Can we write a single lagrangian which allows interpolating between two such spectra with different $q$ ?

A simple example is the following. Consider $\mathrm{U}(1) \times \mathrm{U}(1)$ gauge theory with chiral multiplets $A$ of charge $(1,0), B$ of charge $(-1,0), C$ of charge $(0,1)$ and $D$ of charge $(0,-1)$. We furthermore couple a copy of the chiral spectrum of our last paragraph with two different values of $q$ to the two different U(1)'s. Finally, we write a superpotential which forces the constraint

$$
A B+C D=\mu^{2}
$$

for some scale $\mu$, e.g. by introducing a Lagrange multiplier or squaring the constraint. 
This theory has a moduli space of supersymmetric vacua, and in particular a vacuum in which $A B=0$, the first $\mathrm{U}(1)$ is unbroken and the first chiral spectrum is realized, and another vacuum in which $C D=0$, the second $\mathrm{U}(1)$ is unbroken and the second chiral spectrum is realized.

This already varies the chiral spectrum but does not yet give mass to the chiral multiplets of the "wrong" gauge group. This can be accomplished by additional superpotential terms; for example in the $q=2$ theory we can write

$$
W=\sum_{i} \phi^{(2)}\left(\phi_{i}^{(-1)}\right)^{2}
$$

which allows lifting almost all of the original chiral matter. More complicated superpotentials can tie $\phi^{(2)}$ to $A B C D$ above and accomplish the same thing for $q \neq 2$.

The broken $\mathrm{U}(1)$ is broken at the scale $\mu$, so if this is large, a low energy observer will not see the broken U(1). In examples arising from Kaluza-Klein compactification, this scale will be $1 / R$, the inverse size of the internal space.

The same mechanism could be used for nonabelian gauge groups as well, by postulating nonchiral matter analogous to $A B C D$ sufficient to completely break either gauge group.

A more economical mechanism would only require breaking to a subgroup under which the chiral matter becomes nonchiral. Let us consider $\mathrm{SO}(10)$ grand unified theory containing $N$ spinors $\psi$ in the 16 . Using the coupling $16 \times 16 \times 10$, we can write a coupling to a vector $V_{k}$ as

$$
W=\sum_{m=1}^{N} V_{k} \Gamma_{i j}^{k} \psi_{m}^{i} \psi_{m}^{j}
$$

which can lift all the chiral matter at the cost of breaking to $\mathrm{SO}(9)$. Again, this can be tied to other matter in a way which restores a different $\mathrm{SO}(10)$ elsewhere.

We refrain from going into more detail at this point as there are very few constraints on this problem from effective field theory. The only constraint on possible transitions in general $\mathcal{N}=1$ supersymmetric or non-supersymmetric theories is the rather trivial one that if the total number of Weyl fermions starts off even (resp. odd), it must remain even (resp. odd), as fermions can only be lifted in pairs. If one requires that some part of the gauge group remains unbroken or that some chiral multiplets remain massless along the transition, this could lead to further constraints, but no specific motivation for such conditions comes to mind.

\subsection{Kaluza-Klein reduction on $S^{1}$}

Let us proceed instead by considering specific constructions which arise from higher dimensional field theory or string theory.

The simplest example is the compactification of $d=5$ gauge theory with $\mathrm{SU}(N)$ vector bundle on $S^{1} \times \mathbb{R}^{3,1}$. Although much discussed in the literature and surely familiar to most readers, it is a good warm-up as it already illustrates in what sense the usual concepts of low energy gauge theory suffice to describe the situation, and the sense in which the true gauge group contains infinitely many copies of the low energy gauge group. 
Let the $S^{1}$ have coordinate $x^{5}$ with periodicity $2 \pi$. A solution of the Yang-Mills equation on $S^{1}$ is a flat connection, whose gauge invariant data is a choice of the holonomy $W_{q}=\exp \left\{i \int d x^{5} A_{5}\right\}$ up to conjugation. By applying a gauge transformation in the connected component of the identity one can bring $A_{5}\left(x_{5}\right)$ to a constant connection $A_{5}=$ $\vec{\theta}$, with $\vec{\theta}=\operatorname{diag}\left(\theta_{1}, \theta_{2}, \ldots, \theta_{N}\right)$ and $\sum_{i} \theta_{i}=0$. Permutation of the $\theta_{i}$ are also gauge transformations (the Weyl group). A generic Wilson line will break the gauge group to $\mathrm{U}(1)^{N-1}$.

There are also gauge transformation not continuously connected to the identity, which can shift any $\theta_{i}$ by an integer. Thus the path $\theta_{i}=-\theta_{j}=t$ with $t \in[0,1]$ is a a closed loop in the moduli space, with unbroken $\mathrm{SU}(2)$ symmetry at $t=0,1$ broken to $\mathrm{U}(1)$ at intermediate values.

While in this example the two endpoints are physically equivalent models, there is a clear sense in which the two SU(2) subgroups are different embeddings in a larger, infinite dimensional gauge group, in this case a loop group. Let us see this by doing the KK reduction explicitly. The five dimensional gauge connection decomposes into a spacetime component and an internal space component, $A_{M}=\left(A_{\mu}, A_{5} \equiv \phi\right)$. Choose the internal gauge connection to be flat and diagonal, as above. The Fourier expansion of the spacetime components is

$$
A_{\mu}\left(x^{\mu}, x_{5}\right)=\sum_{n} A_{\mu}^{(n)} e^{-i n x^{5}} ; \quad \phi\left(x^{\mu}, x_{5}\right)=\sum_{n} \phi^{(n)} e^{-i n x^{5}}
$$

$A_{\mu}$ and $\phi$ are hermitian, so $\left(A_{\mu}^{(n)}\right)^{\dagger}=A_{\mu}^{(-n)}$ (resp. $\phi$ ). In term of the matrix element, this is $\left(a_{i j}^{(n)}\right)^{*}=a_{j i}^{(-n)}$. We expand the parameter $\epsilon(x)$ of local gauge transformations in the same way.

The four dimensional lagrangian for the vector bosons is then

$$
\begin{aligned}
\mathcal{L} & =\int_{S_{1}} \operatorname{tr} F_{\mu \nu}^{2}+\operatorname{tr} F_{\mu 5}^{2} \\
\int_{S_{1}} \operatorname{tr} F_{\mu \nu}^{2} & =\sum_{n} F_{\mu \nu}^{(n)} F_{\mu \nu}^{(-n)} \\
\int_{S_{1}} \operatorname{tr} F_{\mu 5}^{2} & =\int_{S_{1}} \operatorname{tr}\left(\partial_{5} A_{\mu}+i\left[\phi, A_{\mu}\right]\right)^{2} \\
& =\sum_{n} \operatorname{Tr}\left[\left(-i n A_{\mu}^{(n)}+i\left[\phi, A_{\mu}^{(n)}\right]\right)\left(i n A_{\mu}^{(-n)}+i\left[\phi, A_{\mu}^{(-n)}\right]\right)\right] \\
& =\sum_{n} \sum_{i, j}\left[\left(\theta_{i}-\theta_{j}\right)-n\right]^{2} a_{i j}^{(n)} a_{j i}^{(-n)} .
\end{aligned}
$$

The $F_{\mu 5}^{2}$ terms provide mass terms for almost all of the low energy gauge bosons. Note that whenever $\theta_{i}-\theta_{j}=n$ is an integer, the non-diagonal gauge bosons $A_{i j}^{(n)}$ become massless, and part of the broken symmetry is restored. A flow from $n$ to $n+1$ will however complete the diagonal $\mathrm{U}(1)$ gauge symmetry (for which the gauge bosons are always $a_{i i}^{(0)}$ ) to $\mathrm{SU}(2)$ using "different" off-diagonal gauge bosons $A_{i j}^{(n)}$ and $A_{i j}^{(n+1)}$. 
In the low energy theory, this is just the standard Higgs phenomenon, as can be seen by considering the gauge transformation:

$$
\phi\left(x^{5}\right) \rightarrow g\left(x^{5}\right)^{-1}\left(\partial_{5}+i \operatorname{ad} \phi\left(x^{5}\right)\right) g\left(x^{5}\right) .
$$

Expanded in modes, this is

$$
\delta \phi_{i j}^{(n)}=\left(n+\theta_{i}-\theta_{j}\right) \epsilon_{i j}^{(n)} .
$$

If the $\theta_{i}$ are small, the $\phi^{(n)}$ transform inhomogeneously under the $n \neq 0$ modes of the gauge symmetry, so they are the Goldstone bosons for the broken gauge symmetry. On the other hand, for general $\theta_{i}$ one can unbreak "different" parts of the gauge group.

Although reduction on $S^{1}$ is not going to lead to chiral fermions, one can still see the sense in which massless matter at the two ends of the loop is "different" as well. The case of adjoint fermions is the same as above, while fundamental fermions would decompose into an infinite set of modes $\psi_{i}^{(n)}$ and $\tilde{\psi}_{i}^{(n)}$ with $\mathrm{U}(1)_{i}$ charge \pm 1 and mass $n-\theta_{i}$. Again, any of these modes can appear as massless matter at a point where non-abelian gauge symmetry is restored.

One can get a more geometric picture of the same result by considering a gauge theory on Dirichlet branes wrapped on $S^{1}$ and performing T-duality, leading to Dirichlet branes at points in the dual $S^{1}$. The non-abelian gauge bosons arise as winding strings, and as we move a D-brane around the dual $S^{1}$, "different" winding strings shrink to zero length. We will use this picture in other examples below.

We put "different" in quotes as the two ends of the paths we are considering are in fact completely equivalent under large gauge transformation, a feature which will not be true in the real examples. In effective field theory, one can get a more illustrative example by adding additional terms to this effective lagrangian which spoil the large gauge symmetry, such as couplings to additional matter of the sort we postulated earlier.

Naively, one might think of the underlying gauge group as a simple infinite product of groups " $\mathcal{G}=\prod_{n \in \mathbb{Z}} G_{n}$," while to describe a given path one might keep two of these groups (say $G_{0}$ and $G_{1}$ to describe $\theta \rightarrow \theta+1$ ). This is not a correct interpretation of what we have described. Rather, the gauge group is a loop group, and the diagonal gauge bosons always sit in the same Cartan subalgebra. Thus we have not described the physics in terms of a product of finite dimensional Lie groups, but as an infinite dimensional Lie group.

One can certainly write a model which realizes one such path in terms of two groups $G_{0} \times G_{1}$, by postulating additional bifundamental matter which breaks down to the diagonal subgroup. However this matter is not present in the underlying KK theory as we have written it. One might try to postulate additional matter in hopes of simplifying the overall description, but it is not obvious to us that this is more natural than working with the loop group.

To some extent, the general picture is similar for any compactification, with the "true" gauge group of the effective gauge theory arising from compactification of Yang-Mills with gauge group $G$ on an internal space $M$ being the group of maps from $M$ to $G$. Unfortunately, it is not easy to get a global picture of this group for $M$ of dimension greater than 2. One furthermore expects larger groups to appear for string scale $M$. The most concrete 
version of this we know about appears in the work of Giveon, Porrati and Rabinovici on $\mathcal{N}=4$ supergravity-super Yang-Mills theories arising from toroidal compactification of the heterotic string [9].

\subsection{Connections on $S^{2}$}

We next discuss $S^{2}$ and $T^{2}$. We will be more interested in $T^{2}$, but the story for $S^{2}$ is simpler and illustrates the essential points. Although $S^{2}$ is not Ricci flat, one can realize gauge theory on this space in string theory, by wrapping branes on a minimal volume cycle with topology $S^{2}$.

These spaces can be given complex structures, to become $\mathbb{C P} \mathbb{P}^{1}$ and $\mathbb{C} / \mathbb{Z}^{2}$ respectively, so both cases can preserve supersymmetry, and both can be discussed in the language of algebraic geometry. We will return to this later. One can also appeal to the fairly complete mathematical analysis of Atiyah and Bott [10], which finds the general solutions to the Yang-Mills equations on Riemann surfaces, and discusses how these sit in the total space of connections. The KK compactification of U(1) theory coupled with gravity on $S^{2}$ is discussed in [11, 12].

One can also do straightforward KK reduction. Let us consider $S^{2}$. On a two dimensional manifold, $\mathrm{U}(1)$ bundles are classified topologically by the first Chern class $c_{1}(V)$, taking values in $H^{2}(M, \mathbb{Z}) \cong \mathbb{Z}$. Physically this is a version of the Dirac quantization condition. On the other hand, there are no topological invariants of an $\mathrm{SU}(N)$ bundle on a simply connected two dimensional manifold.

We fix the rotationally symmetric metric on $S^{2}$ as $d s^{2}=d \theta^{2}+\sin ^{2} \theta d \phi^{2}$, the corresponding volume form is $\omega=\frac{1}{4 \pi} \sin \theta d \theta d \phi$, with $\int_{S^{2}} \omega=1$.

A simple $\mathrm{U}(1)$ connection on $S^{2}$ with $c_{1}(V)=n \in \mathbb{Z}$ and which solves the Yang-Mills equations can be written by postulating two patches, $B_{+} \subset S^{2}$ with $\theta<\pi$ and $B_{-} \subset S^{2}$ with $\theta>0$. The connections in the two patches are

$$
A_{ \pm}(\theta, \phi)=-\frac{n}{2} d \phi \frac{\cos \theta \pm 1}{\sin \theta} .
$$

This connection has $F=n \omega$, so $d * F=0$. We will refer to this line bundle (and, making a slight abuse of language, this connection) as $\mathcal{O}(n)$, for reasons we discuss later.

In fact, all solutions of the Yang-Mills equations on $S^{2}$ for any semisimple $G$ can be written as a direct sum of $\mathrm{U}(1)$ solutions $\mathcal{O}\left(n_{i}\right)$, and thus can be specified by an unordered list of integers $n_{i}$ 13]. For $\mathrm{SU}(N)$, the only constraint on these integers is $\sum_{i} n_{i}=0$. We will speak of such an $\mathrm{SU}(N)$ connection as having "split" into $\mathrm{U}(1)$ connections, and the unordered list of $n_{i}$ as the "splitting type."

As $\mathrm{SU}(N)$ connections, the solutions with $n \neq 0$ are not minima of the Yang-Mills action (which becomes the four dimensional effective potential) but are instead saddle points, with unstable (tachyonic) modes. This can be seen explicitly, or by appealing to the results of [10], who show that the number of unstable modes for such a solution is the index of the Yang-Mills functional at the critical point. For $\mathcal{O}(n) \oplus \mathcal{O}(-n)$ on a genus $g$ Riemann surface, this is $2(2 n-1+g)$. On $T^{2}$, it is easy to see in a T-dual brane description, as we discuss below. 
This instability will not prevent us from using these solutions as simple toy examples of chirality change. After all, the question of whether chirality change is possible in effective field theory does not depend on whether the vacua under discussion are stable or not. When we get to the real examples on Calabi-Yau, we will find that this toy model does properly illustrate the basic phenomenon.

Rather, the question is whether these different solutions are topologically connected and lead to different spectra of chiral fermions in the low energy theory. Now as $\mathrm{U}(1)^{N-1}$ connections, these connections are topologically distinct, and classified by the homotopy class of the transition function in $\pi_{1}\left(\mathrm{U}(1)^{N-1}\right)$ up to Weyl reflection (here $S_{N}$ permutations). However, within the space of $\mathrm{SU}(N)$ connections, any of these connections can be deformed into any other; the transition function is classified by $\pi_{1}(\mathrm{SU}(N)$ which is trivial. On the other hand, since all solutions of Yang-Mills take the form we discussed, all of these deformations must pass through connections which do not solve the Yang-Mills equations.

An example of a path in the space of $\mathrm{SU}(2)$ gauge connections, corresponding to the deformation from $\mathcal{O}(n) \oplus \mathcal{O}(-n)$ to $\mathcal{O}(m) \oplus \mathcal{O}(-m)$ as $t$ varies from 0 to 1 , can be constructed as follows. Because the two gauge connections on the two patches can be subject to arbitrary gauge transformations and their forms are not unique, we start from the intrinsic description of the vector bundle by gauge transition function defined at the intersection of the two hemispheres. Its form is unique up to a constant $\mathrm{SU}(2)$ transformation. This one parameter family of $\mathrm{SU}(2)$ valued map on the equator $S^{1}$ is

$$
\begin{aligned}
g(t ; \phi) & =\left(\begin{array}{cc}
\sin ^{2} \pi t+\cos ^{2} \pi t e^{i n \phi} & -\sin \pi t \cos \pi t\left(1-e^{i n \phi}\right) \\
\sin \pi t \cos \pi t\left(1-e^{-i n \phi}\right) & \sin ^{2} \pi t+\cos ^{2} \pi t e^{-i n \phi}
\end{array}\right), \quad t \in\left[0, \frac{1}{2}\right], \\
& =\left(\begin{array}{cc}
\sin ^{2} \pi t+\cos ^{2} \pi t e^{i m \phi} & -\sin \pi t \cos \pi t\left(1-e^{i m \phi}\right) \\
\sin \pi t \cos \pi t\left(1-e^{-i m \phi}\right) & \sin ^{2} \pi t+\cos ^{2} \pi t e^{-i m \phi}
\end{array}\right), \quad t \in\left[\frac{1}{2}, 1\right] .
\end{aligned}
$$

This is a single-valued function from $S^{1}$ to $\mathrm{SU}(2)$, and satisfies all the requirements: at $t=0$ it is of diagonal form

$$
g(\phi)=\left(\begin{array}{cc}
e^{i n \phi} & 0 \\
0 & e^{-i n \phi}
\end{array}\right),
$$

which corresponds to the vector bundle $\mathcal{O}(n) \oplus \mathcal{O}(-n)$, while at $t=1$ for $\mathcal{O}(m) \oplus \mathcal{O}(-m)$; in the middle $t=1 / 2$ it is identity.

Let us explain in a bit more detail how this seemingly complicated expression is obtained. Start from the identity transition function, a very natural deformation path

$$
g^{\prime}(t ; \phi)=\left(\begin{array}{cc}
\sin \pi t & \cos \pi t e^{i n \phi} \\
-\cos \pi t e^{-i n \phi} & \sin \pi t
\end{array}\right) .
$$

At $t=1 / 2$ this is identity as required. But at $t=0$ this is not quite the right one: it differs from equation (2.5) by a constant $\mathrm{SU}(2)$ rotation. It can be easily fixed by multiplying $g^{\prime}(t ; \phi)$ by a coordinate independent path from identity to this constant matrix

$$
g_{0}(t)=\left(\begin{array}{cc}
\sin \pi t & -\cos \pi t \\
\cos \pi t & \sin \pi t
\end{array}\right) .
$$

This produces the final form of the transition function presented above. 
There are several points worth commenting on.

- This smooth deformation goes through non-diagonal SU(2) matrices, and therefore through non-diagonal connections. This is basically required by the single-value condition of the map on the equator.

- This particular path goes through the trivial bundle $\mathcal{O} \oplus \mathcal{O}$, as the transition function at $t=1 / 2$ is the identity. This fits with the fact that the trivial bundles is generic in the moduli space of the $\operatorname{SL}(2, \mathbb{C})$ vector bundles. On the other hand, the moduli subspace which corresponds to nontrivial splittings has nonzero codimension and is not generic. In particular, any two different splitting types can be connected continuously through the trivial bundle phase as we did above.

- It is possible to go directly from $\mathcal{O}(n) \oplus \mathcal{O}(-n)$ to $\mathcal{O}(m) \oplus \mathcal{O}(-m)$ by combining two such deformation together. Specifically, we can construct the deformation path as

$$
g_{n \rightarrow m}(t ; \phi)=\left(\begin{array}{cc}
\sin \frac{\pi}{2} t & \cos \frac{\pi}{2} t e^{i n \phi} \\
-\cos \frac{\pi}{2} t e^{-i n \phi} & \sin \frac{\pi}{2} t
\end{array}\right) \cdot\left(\begin{array}{cc}
\sin \frac{\pi}{2} t e^{i m \phi} & -\cos \frac{\pi}{2} t \\
\cos \frac{\pi}{2} t & \sin \frac{\pi}{2} t e^{-m \phi}
\end{array}\right), \quad t \in[0,1] .
$$

One can easily check that this is a smooth deformation of the $\mathrm{SU}(2)$ transition functions.

Next we discuss the gauge connections. The gauge connections on the two patches are connected by a gauge transformation at the equator $\theta=\frac{\pi}{2}$ through transition function $g(t ; \phi)$, as $\left.A_{+}\right|_{\theta=\frac{\pi}{2}}-\left.g(t ; \phi) \cdot A_{-}\right|_{\theta=\frac{\pi}{2}} \cdot g(t ; \phi)^{-1}=-\frac{1}{i} d g(t ; \phi) g^{-1}(t, \phi)$. The gauge connections on the two patches $A_{ \pm}$can be changed arbitrarily by gauge transformations. It turns out the "symmetric" choice in which $\left.A_{+}\right|_{\theta=\frac{\pi}{2}}=-\left.A_{-}\right|_{\theta=\frac{\pi}{2}}$ does not give a smooth solution for the connections. But there exists appropriate choice of gauge such that $A_{ \pm}$are smooth in separate patches, which is ensured by the smoothness of $g^{-1} d g$. This can be achieved by choosing a gauge connection in one patch, and use $g(t)$ to obtain the connection in another patch. In particular, one can choose

$$
\begin{aligned}
A_{-}(t, \theta, \phi) & =\left(\begin{array}{cc}
-\frac{n}{2} \cos \pi t & 0 \\
0 & \frac{n}{2} \cos \pi t
\end{array}\right) \frac{\cos \theta+1}{\sin \theta}, \quad t \in\left[0, \frac{1}{2}\right] \\
& =\left(\begin{array}{cc}
-\frac{m}{2} \cos \pi t & 0 \\
0 & \frac{m}{2} \cos \pi t
\end{array}\right) \frac{\cos \theta+1}{\sin \theta}, \quad t \in\left[\frac{1}{2}, 1\right], \\
A_{+}(t, \theta, \phi) & =\frac{n}{2} \cos \pi t K(t ; \phi ; n) \frac{\cos \theta-1}{\sin \theta}, \quad t \in\left[0, \frac{1}{2}\right] \\
& =\frac{n}{2} \cos \pi t K(t ; \phi ; m) \frac{\cos \theta-1}{\sin \theta}, \quad t \in\left[\frac{1}{2}, 1\right]
\end{aligned}
$$

where $K(t ; \pi ; n)$ is a $2 \times 2$ hermitian matrix smooth in $\mathrm{t}$ and $\phi$, whose explicit form is not illuminating and we will ignore its detailed form. It smoothly interpolates from $\operatorname{diag}\left(\frac{n}{2},-\frac{n}{2}\right)$ at $t=0$ to 0 at $t=\frac{1}{2}$ to $\operatorname{diag}\left(\frac{m}{2},-\frac{m}{2}\right)$ at $t=0$. 


\subsection{Fermion zero modes on $S^{2}$ and change of chirality}

The net number of chiral fermion zero modes for a fermion coupling to the connections we just discussed is determined by the index theorem,

$$
\operatorname{index} Q D_{\Sigma}=\frac{1}{2 \pi} \int_{\Sigma} \operatorname{tr}_{Q} F
$$

For example, a charge +1 fermion coupled to the connection $\mathcal{O}(n)$ will have $n$ net positive chirality zero modes.

Of course, for a U(1) connection, this number is a topological invariant. The corresponding net number of zero modes for an $\mathrm{SU}(n)$ connection is also given by (2.13), which is zero, and also invariant.

However, if we look at fermion zero modes charged under one of the U(1) subgroups of $\mathrm{SU}(n)$ into which the connections which solve the Yang-Mills equations split, the net number of these zero modes can change. For the path described above, there is a net change of chiral fermion zero modes, as $t$ varies from 0 to $\frac{1}{2}$ to 1 , the net number of chiral fermions varies from $n$ to 0 to $n+1$ correspondingly.

Let us see if this effect can lead to change of chirality in the low energy theory. Let us take $\mathrm{SU}(2)$ for definiteness. Each of the solutions $\mathcal{O}(n) \oplus \mathcal{O}(-n)$ has (naively) the same unbroken gauge symmetry $\mathrm{U}(1)$ with the same embedding, which we can describe in terms of the infinitesimal gauge transformation

$$
\delta A_{\mu}=\left(\begin{array}{cc}
1 & 0 \\
0 & -1
\end{array}\right) \partial_{\mu} \epsilon .
$$

If we consider fermions in the fundamental representation of $\mathrm{SU}(2)$, the two components couple to $\mathcal{O}(n)$ and $\mathcal{O}(-n)$ and respectively have charges +1 and -1 under the low energy $\mathrm{U}(1)$ gauge group. A fermion in the adjoint of $\mathrm{SU}(2)$ will decompose into one neutral fermion, and two off-diagonal components coupling to $\mathcal{O}(2 n)$ and $\mathcal{O}(-2 n)$ with respective low energy $\mathrm{U}(1)$ charges +2 and -2 (in the same units).

The discussion for the two cases is qualitatively the same, with a correlation between the internal and low energy $\mathrm{U}(1)$ charges, which is preserved under complex conjugation. Because of this, the chiral fermions predicted by (2.13), which might naively be thought to cancel between $\mathcal{O}(n)$ and $\mathcal{O}(-n)$, in fact do not cancel from the point of view of low energy chirality.

Consider the reduction of $5+1$ gauge theory for definiteness, and decompose $\mathrm{SO}(1,5)$ into $\mathrm{SO}(1,3) \times \mathrm{SO}(2)$. A chiral spinor in $d=5+1$ (say a 4-component complex Weyl spinor) reduces to $2_{1} \oplus \overline{2}_{-1}$. Because the $d=6$ spinor is complex, the two terms in this decomposition are not complex conjugate but instead are independent $d=4$ spinors, with correlated chirality in $d=4$ and $d=2$.

We can now apply the index theorem as above to conclude that a $d=6$ Weyl spinor in the fundamental leads to $n$ left-handed $d=4$ spinors with $\mathrm{U}(1)$ charge +1 , and $n$ righthanded $d=4$ spinors with $\mathrm{U}(1)$ charge -1 . Complex conjugating the latter, we obtain a 
chiral spectrum of $2 n$ spinors of charge +1 . (Because the original fermion was complex, these are not CPT conjugate.) Similarly, the adjoint representation leads to $2 n$ left handed spinors of charge +2 .

The case of reduction from ten to eight dimensions is similar. Starting with a MajoranaWeyl ten dimensional spinor, we make the decomposition into $\mathrm{SO}(7,1) \times \mathrm{SO}(2)$ spinors $16=8_{1} \oplus 8_{-1}^{\prime}$, which is different from $d=6$ only in that the original spinor is real, so the two terms in the decomposition are complex conjugate to each other. The rest of the argument is the same, leading to a chiral spectrum in $d=7+1$ with multiplicities half of what we found for $d=3+1$.

These results seemed rather counter-intuitive to us at first so let us discuss some of the objections which may come to mind. First, the two chiralities of spinor in $d=5+1$ and $9+1$ are not complex conjugate, so there is no a priori argument that one must start with a complex representation of the gauge group to get chirality. This allowed us to start with $\mathrm{SU}(2)$ and even the adjoint representation and obtain a chiral spectrum in lower dimensions.

One can also notice that the resulting four dimensional spectra are anomalous. This is no contradiction because the six and ten dimensional theories were also anomalous. Since our arguments are classical, this need not concern us. Interesting examples will of course cancel the higher dimensional anomalies, as will happen in string theory, and then the resulting four dimensional spectrum will be non-anomalous.

Finally, for very skeptical readers, there are brane arguments later which may make this result more believable.

\subsubsection{What is going on}

One can use the result we just obtained to get a four dimensional effective theory in which by varying the vacuum expectation value of a field, the parameter $t$ of our family of connections, one can interpolate between theories with different numbers of U(1) charged chiral multiplets.

According to our previous discussion, this does not contradict the standard wisdom, as long as the initial variation of the field breaks the original U(1) gauge group, and a different $\mathrm{U}(1)$ is restored at the endpoint.

What is confusing, is that it appears that both unbroken U(1)'s at the two ends of the path have the same embedding (2.14) in the $\mathrm{SU}(2)$, and thus should correspond to the same $\mathrm{U}(1)$ gauge group in the effective theory. More precisely, a $\mathrm{U}(1)$ gauge boson in the low energy theory arises from a covariantly constant scalar

$$
D_{i} \Phi=\partial_{i} \Phi+\left[A_{i}, \Phi\right]=0
$$

in the higher dimensional theory as

$$
A_{\mu}(x, \theta, \phi)=A_{\mu}(x) \Phi(\theta, \phi) .
$$

Since the connection $\mathcal{O}(n) \oplus \mathcal{O}(-n)$ can be written in terms of (2.2) as

$$
A_{i}=\left(\begin{array}{cc}
A_{ \pm}(\theta, \phi) & 0 \\
0 & -A_{ \pm}(\theta, \phi)
\end{array}\right)
$$


each of these connections admits a covariantly constant scalar

$$
\Phi(\theta, \phi)=\left(\begin{array}{cc}
1 & 0 \\
0 & -1
\end{array}\right)
$$

which are "the same," leading to a paradox.

The subtlety which resolves this is that to properly compare sections of the two bundles, we must work with a presentation in which the transition functions are the same. However, the section (2.15) is defined using transition functions (2.5) which are different for each $n$.

Since all of these $\mathrm{SU}(2)$ bundles are topologically trivial, there is no need to work with patches and transition functions to describe their sections. Concretely, if we can extend the gauge transformation on the equator to (say) the southern patch, we can use it to convert the sections of the adjoint bundle to functions on the sphere, and compare these.

Explicitly, for the bundle $\mathcal{O}(n) \oplus \mathcal{O}(-n)$, we extend the transition function to the southern patch as

$$
g_{+}(\theta, \phi)=\left(\begin{array}{cc}
\cos ^{2} \theta+\sin ^{2} \theta e^{i n \phi} & -\frac{1}{2} \sin 2 \theta\left(1-e^{i n \phi}\right) \\
\frac{1}{2} \sin 2 \theta\left(1-e^{-i n \phi}\right) \cos ^{2} \theta+\sin ^{2} \theta e^{-i n \phi}
\end{array}\right) .
$$

Then the adjoint scalar zero mode $\Phi$, as a function on the sphere, is

$$
\begin{aligned}
& \Phi(n ; \phi)=\left(\begin{array}{cc}
1 & 0 \\
0 & -1
\end{array}\right), \quad \theta \in\left[0, \frac{\pi}{2}\right], \\
&=\left(\begin{array}{cc}
1-\sin ^{2} 2 \theta(1-\cos n \phi) & \sin 2 \theta\left(1-e^{i n \phi}\right)\left(\cos ^{2} \theta+\sin ^{2} \theta e^{i n \phi}\right) \\
\sin 2 \theta\left(1-e^{i n \phi}\right)\left(\cos ^{2} \theta+\sin ^{2} \theta e^{i n \phi}\right) & -1+\sin ^{2} 2 \theta(1-\cos n \phi)
\end{array}\right), \\
& \theta \in\left[0, \frac{\pi}{2}\right] .
\end{aligned}
$$

Obviously, two such functions $\Phi(n ; \phi)$ and $\Phi(m ; \phi)$ on $S^{2}$, which correspond to zero modes of $\mathcal{O}(n) \oplus \mathcal{O}(-n)$ and $\mathcal{O}(m) \oplus \mathcal{O}(-m)$ respectively, are different. This shows that the zero modes at the two ends of the deformation are different, and so are the $\mathrm{U}(1)$ symmetry groups in the effective theory.

One might worry that this argument depends on the choices of extension. To see that it is independent of such choices, observe that the function $\Phi$ at the southern patch is $g_{+}\left(\begin{array}{cc}1 & 0 \\ 0 & -1\end{array}\right) g_{+}^{-1}$. If $\Phi(n)=\Phi(m)$, then on the southern patch one has

$$
g_{+}(m)\left(\begin{array}{cc}
1 & 0 \\
0 & -1
\end{array}\right) g_{+}(m)^{-1}=g_{+}(n)\left(\begin{array}{cc}
1 & 0 \\
0 & -1
\end{array}\right) g_{+}(n)^{-1},
$$

which requires $g_{+}(n) g_{+}^{-1}(m)$ commutes with $\left(\begin{array}{cc}1 & 0 \\ 0 & -1\end{array}\right)$. This is possible only if it is a diagonal matrix. Now $g_{+}(n) g_{+}^{-1}(m)$ is a $\mathrm{SU}(2)$ matrix interpolating from $\left(\begin{array}{cc}e^{i(n-m) \phi} & 0 \\ 0 & e^{-i(n-m) \phi}\end{array}\right)$ at $\theta=\pi / 2$ to the identity matrix at $\theta=\pi$, as $\theta$ varies from $\pi / 2$ to $\pi$. A diagonal $\mathrm{SU}(2)$ matrix is isomorphic to $\mathrm{U}(1)$ which is topologically $S^{1}$. Since it is impossible to interpolate between two maps $S^{1} \rightarrow S^{1}$ with different winding number, $g_{+}(n ; \theta) g_{+}^{-1}(m ; \theta)$ must have non-diagonal elements at certain $\theta$ values. Hence $\Phi_{n} \neq \Phi_{m}$ follows immediately. 
Thus we have seen in detail how a continuous path between two backgrounds solving the Yang-Mills equations on $S^{2}$ can interpolate between low energy theories with different net numbers of $\mathrm{U}(1)$ charged chiral fermions, again because different $\mathrm{U}(1)$ subgroups of the underlying infinite dimensional gauge group are unbroken. One can proceed using KK reduction to develop the effective field theory description, which is most simply done by describing the vector bundles without using transition functions, to obtain an infinite component field theory similar to what we saw for $S^{1}$.

\subsection{The torus $T^{2}$ and the brane picture}

The story for $T^{2}$ is quite similar. One again obtains a large class of solutions to Yang-Mills as direct sums of line bundles. Now, such a solution is not classified only by the first Chern class, but also has two real parameters (for a flat connection this is of course the Wilson line). The solutions corresponding to each splitting type then form a continuous moduli space, as opposed to the case of $S^{2}$ where the solution corresponding to each splitting type is merely a point. Apart from this difference, all other qualitative features are the same. The moduli spaces corresponding to different splitting types are disconnected as Yang-Mills solutions, but they can be connected through a path via gauge connections that are non-solutions. One again finds that direct sums of U(1) connections with nonzero field strength are (unstable) solutions of Yang-Mills with chiral fermion zero modes, whose number is given by the same formula (2.13). Chirality change is possible because the unbroken subgroup, associated to a covariantly constant scalar $D_{i} \Phi=0$, is different for the different saddle points (they can be written explicitly using theta functions).

The $T^{2}$ picture can be made much more intuitive by realizing the gauge theory on branes and taking advantage of T-duality. For definiteness let us consider type-I string theory with 32 D9-branes compactified on $T^{2}$. The maximal gauge group is $\mathrm{SO}(32)$.

The simplest picture is obtained by T-dualizing a single coordinate, to obtain "type-I' theory" with 32 D8-branes and two O8-planes. The T-duality exchange the Kähler moduli parameter $\tau$ and the complex structure parameter $\rho$, and in general turns a torus with nondiagonal metric into a torus with constant background $B$ field. To avoid complication due to constant $B$ flux, we restrict to orthogonal torus (so the metric is diagonal and $\rho$ is pure imaginary) which is mapped into itself after T-duality.

Let us consider a simple example which embeds a U(1) connection with nontrivial field strength in the $\mathrm{SO}(32)$ gauge group. Assume the field strength takes the form

$$
F=\left(\begin{array}{ccccccc}
0 & k & 0 & 0 & 0 & 0 & \ldots \\
-k & 0 & 0 & 0 & 0 & 0 & \ldots \\
0 & 0 & 0 & k & 0 & 0 & \ldots \\
0 & 0 & -k & 0 & 0 & 0 & \ldots \\
\ldots & \ldots & \ldots & \ldots & \ldots & \ddots &
\end{array}\right) d x \wedge d y
$$

The low energy effective theory is a chiral gauge theory with gauge group $\mathrm{SU}(16) \times \mathrm{U}(1)$, and $k$ copies of chiral fermions in the fundamental representation of $S U 16)$, as follows from the index theorem. 

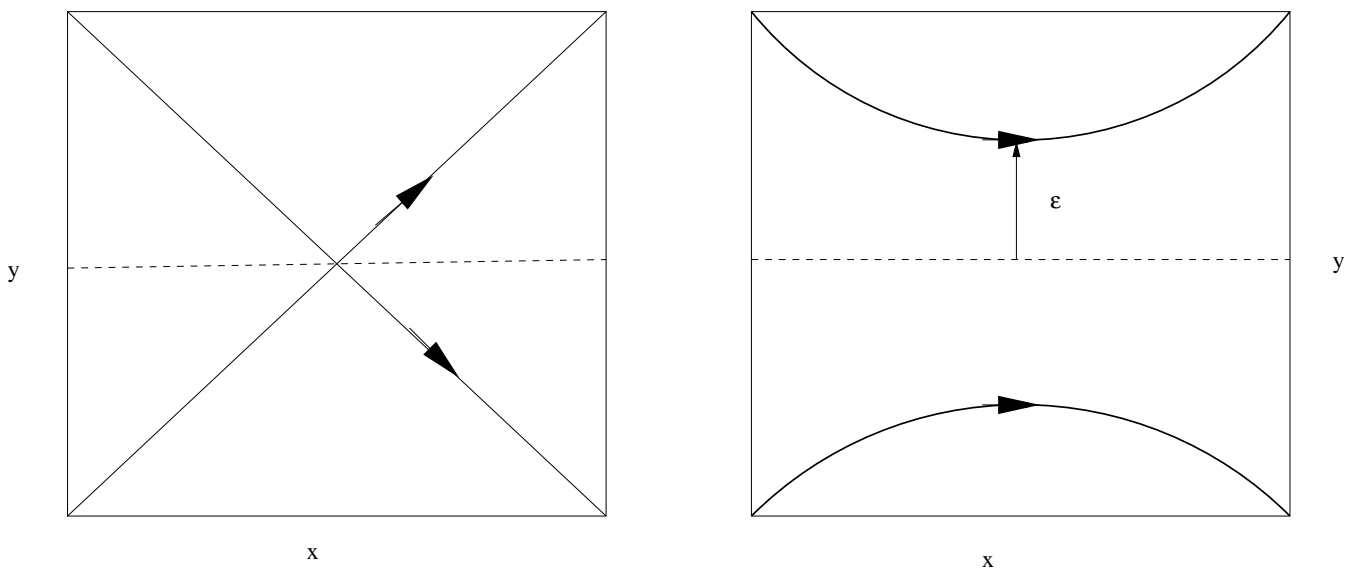

Figure 1: Two intersecting D8 branes deform to a configuration without intersection, which realizes the change of chirality change.

To relate this to our previous discussion, it is simplest to think of $\mathrm{SO}(32)$ as embedded in $\mathrm{SU}(32)$, postulate the $\mathrm{SU}(32)$ connection $\mathcal{O}(k)^{16} \oplus \mathcal{O}(-k)^{16}$, and then impose a reality condition on the fields. This parallels the usual construction of type-I string using the $\Omega$ projection. There is much more to say about this, which we discuss elsewhere.

After T-dualizing in the $y$ direction, the torus is an orbifold $S^{1} / Z_{2} \times S^{1}$. We work in the covering space $T^{2}$. Let $Z_{2}: y \rightarrow-y$, any brane configuration on orbifold is lifted to $Z_{2}$-invariant configuration. The gauge connection in the $y$ direction become the $y$ position of the branes in the dual picture. Thus we have 16 branes described by $y=k x$ and 16 brane images described by $y=-k x$, or in terms of the A- and B-cycles, they wraps $(1, k)$ and $(1,-k)$ respectively. There are $2 k$ intersection points between the two sets of branes, corresponding to $\mathrm{k}$ CPT pairs of chiral fermions in the 16 and $\overline{16}$ representation of SU(16).

A geometric picture of smooth change of chirality from $k=1$ to $k=0$ is shown in figure 1 1. The two sets of branes wrap the one cycle $[a]+[b]$ and $[a]-[b]$ respectively. As one deforms the branes, the chiral fermions living at the two points of intersection of the $k=1$ branes pair up and gain a mass proportional to the distance between the two sets of branes. The intermediate configurations are not minimal surfaces, and the corresponding gauge connections do not satisfy the Yang-Mills equation. Furthermore, they will be irreducible $\mathrm{SU}(2)$ connections, and the gauge group in the effective theory is broken to SU(16). Finally when one reaches a minimal configuration again (all the branes coincide with the O8-branes at one end of $S^{1} / Z_{2}$ ) the gauge group is restored to $\mathrm{SO}(32)$.

Again sice the change of chirality (in the case we just described it is actually disappearance of chiral fermions) is accompanied with the change of gauge group, it can be understood as the Higgs phenomena.

For general $k$ the picture is essentially the same. We see that there are an infinite number of brane configurations, parameterized by $k \in Z^{+} \cup\{0\}$ and each carries $2 k$ chiral fermions, each connected by brane deformation. There are even more configurations, constructed by wrapping branes about the cycles $n[a]+k[b]$. For $g . c . d(n, k)=1$, these are T-dual to irreducible connectionis with rank greater than one [14]. 
In this example, the infinite number of possibilities is clearly related to the fact that we are considering unstable configurations. The only consistent stable configurations are those in which all branes are parallel, which prevents the type of cancellations in the tadpole condition we are seeing here, and it is easy to see that these are finite in number. One hopes that this finiteness is true in higher dimensions as well.

\subsection{Holomorphic bundles, splitting type, and Yang-Mills connections}

To get a similar understanding in Calabi-Yau compactification, we will need better mathematical technology. Most of our understanding in this case is for the special case of supersymmetric compactification of string theory, for which one can appeal to algebraic geometry and the theory of holomorphic bundles.

Within the set of all vector bundles on a complex manifold, one can distinguish the holomorphic bundles, those for which (in some basis) the transition functions are holomorphic. The necessary condition for this is $F^{(0,2)}=0$, so in one complex dimension any vector bundle can be treated this way.

This is a great mathematical advantage as the problem of finding holomorphic bundles does not require solving differential equations; it is purely algebraic. One can then go on to solve the Yang-Mills equations $D * F^{(1,1)}=0$. To be a bit more precise, for holomorphic bundles the natural concept of gauge equivalence is more general; one allows general gauge transformations $g(z) \in G L(N, \mathbb{C}$ ) (or more generally a non-compact group obtained from the complexification of the original Lie algebra), and the statement is that within this larger gauge orbit we can look for specific unitary connections which solve the Yang-Mills equations, here equivalent to $F^{(1,1)}=c \omega$. When one exists, it is unique up to standard (unitary) gauge equivalence; the necessary and sufficient condition for it to exist is $\mu$ stability, as discussed in great detail in [15, 16].

A rank 1 holomorphic bundle is usually called a line bundle. The line bundles on $\mathbb{C P}^{1}$ are classified by the integer $n=c_{1}(V)$ (the degree), and can be written in terms of two patches and a transition function $g=z^{n}$. The usual notation for such a line bundle is $\mathcal{O}(n)$. One can equivalently say that $\mathcal{O}_{\mathbb{C P}^{d}}(n)$ for $n \geq 0$ has as holomorphic sections functions of degree $n$ in the homogeneous coordinates on $\mathbb{C P}^{d}$. The bundles with $n<0$ can be defined as the duals of these, or as bundles whose sections must have poles of total order $-n$.

As for $G L(N)$ bundles on $\mathbb{C P}^{1}$, these are classified by Grothendieck's theorem: a holomorphic vector bundle $V$ can always be written in as a direct sums of line bundles,

$$
V=\oplus_{i} \mathcal{O}\left(n_{i}\right)
$$

where the $n_{i}$ are uniquely determined (again as an unordered list). One sometimes says that the bundle "splits," and this decomposition is its "splitting type." Note that this is a finer classification than by topological type, and in fact is the same as our classification of solutions of the Yang-Mills equations, for reasons we will explain shortly. The theorem also classifies $\operatorname{SL}(N)$ bundles: these are just the subset with $\sum_{i} n_{i}=0$.

As we said, on $S^{2}$ (or any two dimensional compact manifold) all $\operatorname{SL}(N)$ bundles are continuously connected and are all equivalent to holomorphic bundles. Thus it must be 
possible to make continuous transitions between elements of this discrete set of bundles. This seeming paradox is not hard to understand. Consider a family of holomorphic bundles obtained by varying a complex parameter $\alpha$, defined by the transition functions [17, 18

$$
g(z)=\left(\begin{array}{cc}
z & \alpha \\
0 & z^{-1}
\end{array}\right) .
$$

For $\alpha=0$ this is $\mathcal{O}(1) \oplus \mathcal{O}(-1)$. On the other hand, for any $\alpha \neq 0$ it turns out that this is equivalent as a holomorphic bundle to $\mathcal{O} \oplus \mathcal{O}$. This can be seen from the decomposition

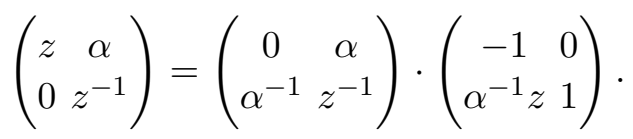

The two matrices can be extended holomorphically to the unit disk centered around $z=0$ and $z=\infty$ respectively. The transition function on the equator is trivial up to gauge transformation on the two open patches, thus corresponds to trivial splitting types of the line bundles.

This phenomenon is generic and is called "jumping". A simple example of a compact family of bundles which exhibits it can be constructed by considering a particular nonuniform rank-2 vector bundle $E$ on $P^{2}$ 15. Since $P^{1}$ can be embedded as a line in $P^{2}$, in a family parameterized by the Grassmannian manifold $G\left(P^{2}, P^{1}\right)$, the pullback of $E$ to $P^{1}$ of embedded yields a family of rank 2 vector bundles on $P^{1}$, whose spitting type over the moduli space $G\left(P^{2}, P^{1}\right)$ demonstrates the jump of degree. More explicitly, blow up $m$ points $x_{1}, \ldots, x_{m}$ on $P^{2}$ to get $Y$ with projection $\sigma: Y \rightarrow P^{2}$. On $Y$, construct a rank two vector bundle $E^{\prime}$ via extension

$$
0 \rightarrow[C] \rightarrow E^{\prime} \rightarrow[-C] \rightarrow 0 .
$$

where $C$ is the exceptional divisors from the blown up. It can be proved that $E^{\prime}$ actually is a lift of a rank two vector bundle $E$ over $P^{2}, E^{\prime}=\sigma^{*} E$. $E^{\prime}$ restricted to the exceptional divisor $C_{i}$ are of the form

$$
0 \rightarrow \mathcal{O}_{C_{i}}(-1) \rightarrow \mathcal{O}_{C_{i}} \oplus \mathcal{O}_{C_{i}} \rightarrow \mathcal{O}_{C_{i}}(1) \rightarrow 0
$$

Because these exceptional curves become points in $P^{2}$, when the line $P^{1}$ pass through any point $x_{i}$, we expect a splitting of $\mathrm{E}$ as $\mathcal{O} \oplus \mathcal{O} \rightarrow \mathcal{O}(1) \oplus \mathcal{O}(-1)$. This is indeed what happens. The general theorem states that the restriction of the rank 2 vector bundle $\mathrm{E}$ to any line $L$, on which exactly $\mathrm{k}$ points of the set $\left\{x_{1}, \ldots, x_{m}\right\}$ lie, splits in the form

$$
\left.E\right|_{L}=\mathcal{O}_{L}(k) \oplus \mathcal{O}_{L}(-k) .
$$

The generic splitting type of this bundle is $\mathcal{O}_{P^{1}} \oplus \mathcal{O}_{P^{1}}$.

Any rank one line bundle on $\mathbb{C P}^{1}$ is $\mu$-stable and thus admits connections solving the Yang-Mills equations, (2.2). Thus, all of the considerations of the previous sections using explicit gauge connections can be translated to this holomorphic context. We will see some of this in a Calabi-Yau example below. 
To avoid confusion, one should keep in mind that despite the fact that the holomorphic bundles of different splitting type are connected by an infinitesimal variation of parameter $\alpha$, the corresponding solutions of the Yang-Mills equations are separated by a finite distance. We saw this explicitly in the previous, non-algebraic geometry analysis. In general, a family of solutions of hermitian Yang-Mills, only arises from a family of holomorphic bundles.

\section{Chirality change on compact Calabi-Yau in heterotic string theory}

Having understood chirality change in two dimensions, we proceed to six-dimensional compact Calabi-Yau manifold, keeping in mind the idea that isomorphic gauge groups can have different embeddings in the same yet higher dimensional gauge group.

By now the reader is probably willing to grant the argument we gave in the introduction, that since $c_{3}(V)$ is not a topological invariant of $E_{8} \times E_{8}$ or $\operatorname{Spin}(32) / \mathbb{Z}_{2}$ bundles, there is no topological barrier to changing the number of generations. We still need to check that in fact there are compactifications on the same CY with different numbers of generations which could be connected in this way.

To find such supersymmetric compactifications, we just need to find examples of semistable holomorphic bundles on the same CY whose Chern classes differ only in $c_{3}$. This is more or less already known from the study of $(0,2)$ models and is in any case easy to show using the techniques commonly used there, as we will do.

We will then proceed to look for something stronger - a path between two vacua with different numbers of generations completely through supersymmetric configurations.

\subsection{Chirality from reducible connections}

Consider heterotic string theory with gauge group $E_{8} \times E_{8}$ compactified on a simply connected compact Calabi-Yau manifold $M$, and assume the gauge connection on Calabi-Yau is embedded in the first $E_{8}$ group. $\mathcal{N}=1$ supersymmetry in spacetime effective theory then requires the following:

1. The Bianchi identity for the 3 -form field strength $H$ of the 2-form $B$ field $d H=$ $\operatorname{tr} R \wedge R-\operatorname{tr} F \wedge F$ requires that vector bundle $W$ and the tangent bundle of the Calabi-Yau manifold $T M$ to have same second Chern classes. More generally, this equation also receives a five-brane contribution, but we will not use this.

2. The gauge connection $A$ on vector bundle $W$ should satisfy the hermitean-Yang-Mills equation, $F_{\bar{a} \bar{b}}=F_{a b}=0$ and $g^{a \bar{b}} F_{a \bar{b}}=0$. By the Donaldson-Uhlenbeck-Yau theorems, such a solution exists if and only if the vector bundle $W$ carrying representation of $A$ is a direct sum of stable bundles with slope zero granting $c_{1}(W)=0$ (for more on this see the appendix).

Note that the topological type of $W$ as an $E_{8}$ bundle is completely fixed by $c_{2}(W)$, which we fixed in (1). This is because the homotopy groups $\pi_{i}\left(E_{8}\right)$ vanish for all $i \neq 3$ and $i \leq 6$ (in fact, for all $i \leq 14$ ). Thus all such compactifications are connected, in general through non-supersymmetric configurations. 
Of course, compactification with an $E_{8}$ connection leaves no chiral fermions (and no unbroken gauge group) perturbatively. To get a non-trivial low energy gauge group $G$, we choose a subgroup $H \subset E_{8}$ and a bundle $V$ with structure group $H$; $G$ is then the commutant of $H$ in $E_{8}$. The moduli space of such $V$ is a subset of the total moduli space of bundles $W$, and in the neighborhood of $V$ one can think of this condition as simply setting the $G$-charged fields to zero.

$W$ can then be decomposed into a direct sum of vector bundles carrying representations of $G \times H$, and one can then use the index theorem to find the chiral spectrum.

The index theorem for a Dirac operator taking values in a representation $Q$ of the gauge connections on a six-dimensional manifold $\mathrm{X}$ is

$$
\operatorname{index}_{Q} \not D_{X}=\frac{1}{48(2 \pi)^{2}} \int_{X}\left[\operatorname{tr}_{Q} F \wedge F \wedge F-\frac{1}{8} \operatorname{tr}_{Q} F \wedge \operatorname{tr} R \wedge R\right]
$$

For a vector bundle with $c_{1}=0$, as will be the case for $H=\mathrm{SU}(k)$, and the fundamental representation (as for GUT matter in the 27,16 , or 10 with $k=3,4,5$ ), this reduces to the third Chern class of the gauge bundle, and we find $N_{\text {gen }}=c_{3}(V) / 2$.

We repeat this well-known material mostly to make the point that there is a direct analogy between $c_{3}(V)$ in this construction, and a quantity such as $k$ in $O(k) \oplus O(-k)$ in our toy models. It is not a topological invariant of the $E_{8}$ bundle, but rather is directly analogous to the idea of "splitting type" we discussed earlier.

\subsection{Finiteness of the number of possible $c_{3}$ 's}

A very central question in superstring compactification is whether there are finitely or infinitely many possible compactifications which might be candidates to describe our universe. At present even very basic questions of this type remain unanswered; for example whether or not the number of Calabi-Yau threefolds is finite.

A question of this type for which the answer is actually known is whether the $\mathrm{SU}(k)$ bundles which satisfy the above requirements for heterotic string compactification, come with a finite or infinite number of distinct values of $c_{3}(V)$. In fact, there is a finite list of possible values. ${ }^{1}$ This follows for a theorem of Maruyama [21], and more recent work of Langer [22], which show that, having fixed the rank, $c_{1}$ and $c_{2}$, there are a finite number of possible values of $c_{3}$ taken by semistable bundles.

An intuitive explanation of the result is the following. One considers a hypersurface $\Sigma$ in the CY3, and considers the restriction of $V$ to $\Sigma$. One can show that, for sufficiently high degree $\Sigma$, distinct bundles $V_{1}$ and $V_{2}$ must restrict to distinct bundles on $\Sigma$ (this follows from considering the exact sequence governing the restriction of $\operatorname{Hom}\left(V_{i}, V_{j}\right)$ to $\Sigma$ ), so the restriction actually determines the original bundle on $V$. Next, note that on restriction, the bundle on $\Sigma$ can inherit all of the information in the rank, $c_{1}$ and $c_{2}$, but loses all knowledge of $c_{3}$. Finally, there is a general result called "algebraicity of the family," which states that a family of bundles for which all Chern classes are specified is algebraic, roughly meaning that its moduli space can be defined by a finite system of equations and thus consists of a

\footnotetext{
${ }^{1}$ M.R.D. thanks F. Bogomolov and A. Langer for explanations on the content of this subsection.
} 
finite set of branches. Thus, the combined moduli space of all bundles with all values of $c_{3}$ consists of a finite set of branches, and thus there must be a finite number of possible values of $c_{3}$.

Unfortunately, there is no effective version of this theorem, which gives actual bounds on the values of $c_{3}$ which can be attained.

\subsection{Explicit search on the quintic}

We first note that we can construct sheaves on the ambient projective space, and restrict these to the quintic. This does not give us all bundles; for a start it imposes conditions on the Chern classes. For example, on the quintic, we will only get $c_{3}(V)$ divisible by five. Furthermore, we search only the class of vector bundles produced by the two term monad construction on weighted projective space. There is a more general three term monad which is the general class of bundle produced by the linear sigma model. More general complexes are possible as well; on general grounds one expects to need at least four terms to get all bundles.

We start a bit more generally by considering intersections of hypersurfaces in weighted projective space. If we do not need to resolve singularities, the resulting even cohomology space has $\operatorname{dim} H^{1,1}(M)=\operatorname{dim} H^{2,2}(M)=1$, so the anomaly cancellation condition imposes a minimal constraint. The bundles we are seeking satisfy conditions

$$
c_{1}(V)=0, \quad c_{2}(V)=c_{2}(T M), \quad c_{3}(V) \text { arbitrary } .
$$

The monad construction of vector bundles is studied in [23]-[27].

Weighted projective space $C P_{w}^{N+3}$ has homogeneous coordinates $\left(X_{1}, X_{2}, \ldots, X_{N+3}\right)$ with weights $w=\left(w_{0}, w_{1}, \ldots, w_{N+3}\right)$ under a $\mathrm{U}(1)$ action. A 3 -fold is defined as zero locus of $\mathrm{N}$ polynomials $P_{1}(X), P_{2}(X), \ldots, P_{N}(X)$ with degrees $p=\left(p_{1}, p_{2}, \ldots, p_{N}\right)$. The Calabi-Yau condition requires the first Chern class to be zero,

$$
c_{1}(T X)=\left(\sum_{i=0}^{N+3} w_{i}-\sum_{j=1}^{N} p_{j}\right) J,
$$

where $J=c_{1}(\mathcal{O}(1))$ is the first Chern class of the hyperplane bundle on the projective space. The Calabi-Yau condition requires $c_{1}(T X)=0$, so

$$
\sum_{i=0}^{N+3} w_{i}=\sum_{j=1}^{N} p_{j}
$$

Using this condition, the second and the third Chern class can be expressed as

$$
\begin{aligned}
& c_{2}(T X)=\frac{1}{2}\left(\sum_{j=1}^{N} p_{j}^{2}-\sum_{i=0}^{N+3} w_{i}^{2}\right) J^{2}, \\
& c_{3}(T X)=\frac{1}{3}\left(\sum_{i=0}^{N+3} w_{i}^{3}-\sum_{j=1}^{N} p_{j}^{3}\right) J^{3} .
\end{aligned}
$$


The monad construction of rank-n vector bundle $\mathrm{V}$ is defined by a short exact sequence

$$
0 \rightarrow V \rightarrow \oplus_{i=1}^{n+M} \mathcal{O}\left(n_{i}\right) \stackrel{\phi=\left(Q_{j}^{i}(X)\right)}{\longrightarrow} \oplus_{j=1}^{M} \mathcal{O}\left(m_{j}\right) \rightarrow 0 .
$$

where the map $\phi$ is defined by a $M \times(M+n)$ matrix of polynomials $Q_{j}^{i}\left(X_{1}, X_{2}, \cdots, X_{N+3}\right)$ $(i=1, \ldots, M+n ; j=1, \ldots, M)$ with degree $\operatorname{deg}\left(Q_{j}^{i}\right)=m_{j}-n_{i}$. Obviously,

$$
m_{j}>n_{i}>0, \quad \forall i, j
$$

otherwise the corresponding polynomial is zero. Notice that the degree is in terms of $\mathcal{O}(1)$, the hypersurface on $C P_{W}^{N+3}$.

The first Chern class for such a bundle is

$$
c_{1}(V)=\left(\sum_{i=0}^{n+M} n_{i}-\sum_{j=1}^{M} m_{j}\right) J .
$$

One of the necessary condition for semi-stability is $c_{1}(V)=0$, which requires

$$
\sum_{i=0}^{n+M} n_{i}=\sum_{j=1}^{M} m_{j}
$$

Using this condition, the second and the third Chern classes of V can be expressed as

$$
c_{2}(V)=\frac{1}{2}\left(\sum_{j=1}^{M} m_{j}^{2}-\sum_{i=0}^{n+M} n_{i}^{2}\right) J^{2}, \quad c_{3}=\frac{1}{3}\left(\sum_{i=0}^{n+M} n_{i}^{3}-\sum_{j=1}^{M} m_{j}^{3}\right) J^{3} .
$$

To conclude, on a Calabi-Yau space $M$ defined as intersection of hypersurfaces in the weighted projective space specified by weights $w_{a}(a=1, \ldots, N+3)$ and degree of polynomials $p_{b}(b=1, \ldots, N)$, define a constant

$$
B=\sum_{j=1}^{N} p_{j}^{2}-\sum_{i=0}^{N+3} w_{i}^{2}
$$

such that the second Chern class of the tangent bundle is $c_{2}(T M)=\frac{1}{2} B \cdot J^{2}$. The rank-n vector bundle $\mathrm{V}$ is specified by two sets of integers $\left(n_{i} ; m_{j}\right)$ constrained by

$$
\sum_{i=0}^{n+M} n_{i}=\sum_{j=1}^{M} m_{j}, \quad \sum_{i=0}^{n+M} n_{i}^{2}+B=\sum_{j=1}^{M} m_{j}^{2}, \quad m_{j}>n_{i}>0, \quad \forall i, j .
$$

After solving these constraints one can calculate the corresponding $c_{3}(V)$.

First, there exists at least one solution to such sets of constraints, namely, the tangent bundle of the Calabi-Yau manifold itself. The next qualitative question is whether the number of solutions to these constraints is finite or infinite. In fact, as we argue in appendix B, the number of solutions to these constraints is always finite. 


\begin{tabular}{|c|c|c|}
\hline rank $(\mathrm{n})$ & $\left(n_{i}, m_{j}\right)$ & $N_{\text {gen }}$ \\
\hline \hline 3 & $(22222222 ; 33334)$ & 90 \\
\hline 3 & $(122222 ; 344)$ & 95 \\
\hline 3 & $(112233 ; 444)$ & 100 \\
\hline 3 & $(11222 ; 35)$ & 105 \\
\hline 3 & $(11133 ; 45)$ & 110 \\
\hline 4 & $(1122222222 ; 333333)$ & 80 \\
\hline 4 & $(11122222 ; 3334)$ & 85 \\
\hline 4 & $(111122 ; 44)$ & 90 \\
\hline 4 & $(11111 ; 5)$ & 100 \\
\hline 5 & $(1111122222 ; 33333)$ & 75 \\
\hline 5 & $(11111122 ; 334)$ & 80 \\
\hline 6 & $(1111111122 ; 3333)$ & 70 \\
\hline 6 & $(111111111 ; 234)$ & 75 \\
\hline 7 & $(11111111111 ; 2333)$ & 65 \\
\hline 7 & $(111111111111 ; 22224)$ & 70 \\
\hline 8 & $(11111111111111 ; 222233)$ & 60 \\
\hline
\end{tabular}

Table 1: List of holomorphic vector bundles on quintic Calabi-Yau manifold with $c_{1}=0, c_{2}=$ $c_{2}(T M)$. They are found using monad construction.

We performed an exhaustive search for the case of the quintic hypersurface for data solving the above constraint equations. and rank up to 8 . The result is shown in table 1 . The number of generations is given by $N_{\text {gen }}=\frac{1}{2} C_{3}(V)$.

The tangent bundle is the entry with rank 3 and $N_{\text {gen }}=100$. In all, there are five rank-3 and four rank-4 holomorphic vector bundles that satisfy all the constraints yet yield different $c_{3}$. Notice that there are several vector bundles of different ranks yet with the same Chern classes. It is possible that taking the direct sum of trivial line bundles with the lower rank vector bundle and defroming it results in the high rank one.

In general, the monad construction leads to coherent sheaves, not vector bundles: they have singularities where the rank of the bundle at that point is smaller than the generic rank, which happens when the dimension of the kernel of the map $\phi$ in (3.7) changes. It is not in general known which singularities are allowed in the perturbative heterotic string, so to be sure of having examples, we should check that some of these can be bundles.

Further, we need supersymmetric vacua. This is equivalent to require the holomorphic vector bundle to be (semi-)stable. In general, it is not easy to verify the (semi-)stability of a given holomorphic vector bundle on a compact manifold, especially when the rank is larger that two. One must find all the holomorphic subsheaves, and then compare the slopes $c_{1}(V) / \operatorname{rank}(V)$.

In appendix A, we do both checks for the holomorphic vector bundles $V_{(1,1,1,1,2,2 ; 4,4)}$ on the quintic. This case is interesting bcause it has the same rank as the direct sum of holomorphic tangent bundle with the trivial line bundle on the quintic, yet yields a 
different number of chiral fermions. We indeed prove these include vector bundles, but check stability only against subsheaves that have a similar monad description, i.e. as a kernel of a map between direct sum of line bundles on $P^{4}$. It would be nice to complete this proof of stability.

Thus there are different embeddings of the $\mathrm{SO}(10)$ gauge group in $E_{8}$ with different number of generations. By our general arguments, these two theories are connected by passing through nonsupersymmetric vacua. The question of whether they can be connected through supersymmetric vacua appears to depend on the precise type of singularity we allow in the bundle; we leave this for future work.

\section{Smooth chirality change in type-IIA orientifold}

In this section, we will give an explicit example in the context of type-IIA orientifold compactification with D6 branes intersecting at angles. These also give string vacua with $D=4 \mathcal{N}=1$ together with various gauge groups and chiral fermions. We construct examples of chirality change through supersymmetric deformation of brane configurations. The geometric picture makes the process more explicit. The formalism goes as follows. The topological constraint satisfied by the homology classes can be realized by different set of branes wrapping lagrangian 3-cycles. Isomorphic effective theory gauge groups can appear with different content of chiral fermions, naturally arises as different microscopic brane wrapping in the internal space.

We first briefly review the general setup of type-IIA orientifold compactification, in particular pay attention to the supersymmetric condition of brane configuration. Then we construct an explicit example, in the case of $T^{6} / Z_{2} \times Z_{2}$, of string theory vacua with isomorphic gauge groups yet different number of chiral multiplets. Further, by a chain of supersymmetric deformations, we are able to prove that they are smoothly connected. Finally, we compare with the heterotic string compactification.

\subsection{Type-IIA orientifold compactification on $T^{6} / \mathbb{Z}_{2} \times \mathbb{Z}_{2}$}

Type-IIA compactification on orientifolds has attracted a lot of attention recently. The anomaly cancellation requires the existence of background D-branes to cancel the negative tension and the R-R charge carried by the orientifold planes. Early work in $D=6$ compactification see [28, 29]. This results in the so-called brane-world theory, where both gauge fields and charged chiral multiplets are present, and, other than the traditional heterotic/type-I string compactification on Calabi-Yau, provides another way to realize string vacuum with phenomenology interests.

In this setup, choose the orientifold $Z_{2}$ action to be complex conjugation on the internal Calabi-Yau 3-fold, the orientifold plane is a real dimension three special lagrangian submanifold. D6 branes wrapping special lagrangian submanifold of the internal CalabiYau while extending in all four noncompact dimensions, results in string theory vacua with $d=4 \mathcal{N}=1$ supersymmetry. If all the D-branes lie within the orientifold planes, then only symplectic groups appear. However, supersymmetry allows D-branes to intersect with angles, then unitary groups can be obtained and chiral fermions [30, 31] are 
supported at the intersection points, finally standard model like spectrum can be obtained. The simplest geometry allowing chiral theory is $T^{6} / \mathbb{Z}_{2} \times \mathbb{Z}_{2}$. Some of the recent discussions on general orientifold of the Calabi-Yau manifold can be found in [32][39]. Actually the anomaly cancellations conditions allow many solutions, with various gauge groups and charged matters. This class of models has been exploited extensively in an effort to find the standard-model-like spectrum for phenomenological model building (see 40, 41]).

In the following, we will concentrate on the orientifold $T^{6} / \mathbb{Z}_{2} \times \mathbb{Z}_{2}$. The orientifold group is generated by three mutually commutative order 2 group elements, $\{\Omega R, \theta, \omega\} . \theta$ and $\omega$ generate $\mathbb{Z}_{2} \times \mathbb{Z}_{2}$ orbifold group, $\Omega$ is a world-sheet parity transformation while $\mathrm{R}$ is a spacetime symmetry acting as complex conjugation on all three complex coordinates and reversing the orientation. Use complex variables $\left(z_{1}, z_{2}, z_{3}\right)$ to parameterize the tori, these generators act as follows

$$
\begin{aligned}
R: & \left(z_{1}, z_{2}, z_{3}\right) \rightarrow\left(\bar{z}_{1}, \bar{z}_{2}, \bar{z}_{3}\right), \\
\theta: & \left(z_{1}, z_{2}, z_{3}\right) \rightarrow\left(-z_{1},-z_{2}, z_{3}\right), \\
\omega:\left(z_{1}, z_{2}, z_{3}\right) & \rightarrow\left(z_{1},-z_{2},-z_{3}\right) .
\end{aligned}
$$

Quite differently from the two generators of the orbifold group which preserve the complex structure of the tori, $\Omega R$ is non-holomorphic and, together with the orientation reversion on the string worldsheet, it reduces the amount of supersymmetry into half. When lift to the action on the Chan-Paton factors from the intersecting branes $(\Omega)$, it requires a projection on the gauge bundle that amount to a real (pseudoreal) structure and results in SO and SP gauge groups when the branes coincide with the orientifold planes. Notice that each element of the form $\Omega R g$ for $g \in \mathbb{Z}_{2} \times \mathbb{Z}_{2}$ is of order 2 , so the fixed hyperplane under these elements are all special lagrangian submanifolds.

The orientifold string theory background has exact conformal field theory description, despite the presence of the singularities of the fixed tori and 64 fixed points. It is a particular point of the moduli space of Calabi-Yau space, viewed as a fibration of K3 space over $P^{1}$. As shown in [42], without discrete torsion, it belongs to a family of Calabi-Yau manifolds with $h^{1,1}=51$ and $h^{2,1}=3$. Turning on discrete torsion, it can be deformed into smooth Calabi-Yau manifold with $h^{1,1}=3$ and $h^{2,1}=51$ which is actually the mirror of the Calabi-Yau without the discrete torsion. For simplicity, we will fix the complex moduli space of the Calabi-Yau moduli space at point where $T^{6}$ is a direct product of complex 2 -tori, each fixed at modulus $\tau=i$ (so the lattice is rectangular). The discussion will be similar for other complex moduli. Throughout this section, we will be interested only in the open string moduli from the intersecting D6 branes.

The consistent condition for string vacua on the open string sector is purely topological, just as the constraint on the second Chern class in the heterotic/type-I case. As usual, this can be understood from both worldsheet and the spacetime point of view. From string worldsheet, there is untwisted RR tadpole. Its cancellation requires the charges of D6branes and O6-branes to cancel [29, 30, 31. From spacetime point of view, orientifold fixed planes carries negative tension and $R R$ charges, the latter being the source of the $R R$ 
7-form. In a compact space, the total charge have to be zero, which requires to add D6 branes to cancel. The twisted tadpole can be cancelled by choosing appropriate projective representations of orientifold group action on the gauge bundle formed by the overlapping D-branes, as in [8].

All D6 and $\mathrm{O} 6$ branes wrap 3-cycles of $T^{6} /\left(Z_{2} \times Z_{2}\right)$. By supersymmetry, these are calibrated submanifold with respect to the holomorphic 3-forms of the Calabi-Yau space: $\left.\omega\right|_{C}=0,\left.\operatorname{Re} \Omega\right|_{C}=\left.V\right|_{C}$. Here $\omega$ is the Kahler form, and $\Omega$ is the holomorphic 3-form, not to be confused with the orientifold action. By definition, these are special lagrangian submanifold. And all span the noncompact four dimensional spacetime. In the following, we will discuss the brane configuration in the covering space $T^{6}$. Our example in the following sections will explicitly contain branes in groups of 4 , so one does not need to worry about whether the branes involved are fractional and have to be stuck to the fixed point/tori of the orbifold group. On the other hand, the complex conjugation $R$ is different from element of $\mathbb{Z}_{2} \times \mathbb{Z}_{2}$, in that the Calabi-Yau space is not quotient by $R$, but merely the $\Omega R$ image of each brane is required to be explicitly included in the background, for example, in the counting of the homology classes of the D-branes. But in any case, our example has all the brane images under the whole orientifold group action.

One of the easiest brane configuration is that they are in factorized form: each brane is direct product of three circles, each is a one cycle on the three tori. However, it is important to realize that these are not the only supersymmetry configuration, which will be the basis for our discussion of the brane deformation in the section below. We will fix some notation here. Let the two fundamental one-cycles of the i-th tori be $\left[a_{i}\right]$ and $\left[b_{i}\right]$ respectively. Under this basis, any one-cycle on the $\mathrm{i}$-th torus is $n^{i}\left[a_{i}\right]+m^{i}\left[b_{i}\right]$, specified by the winding number $\left(n^{i}, m^{i}\right)$. Thus a SLAG with the factorized form has corresponding homology class

$$
[\Pi]=\Pi_{i=1}^{3}\left(n^{i}\left[a_{i}\right]+m^{i}\left[b_{i}\right]\right) .
$$

Its $\Omega R$ image has homology class represented by

$$
\left[\Pi^{\prime}\right]=\Pi_{i=1}^{3}\left(n^{i}\left[a_{i}\right]-m^{i}\left[b_{i}\right]\right),
$$

since $R$ reverses the direction of the b-cycles, while a-cycles remain invariant. Now one can easily specify the RR tadpole cancellation condition. For k stacks of D6 branes labelled by $a=1, \ldots, k$, each stack having $N_{a}$ branes wrapping a factorized special lagrangian submanifold with winding number $\left(n_{a}^{i}, m_{a}^{i}\right)$, one has

$$
\sum_{a} N_{a}\left[\Pi_{a}\right]+\sum_{a} N_{a}\left[\Pi_{a}^{\prime}\right]+(-4) \times 8\left[\Pi_{O 6}\right]=0 .
$$

Notice that the orientifold branes carry -4 charge each, and $\left[\Pi_{O 6}\right]=\left[\Pi_{\Omega R}\right]+\left[\Pi_{\Omega R \theta}\right]+$ $\left[\Pi_{\Omega R \omega}\right]+\left[\Pi_{\Omega R \theta \omega}\right.$ is the total contribution of all the 3-cycles fixed under group element involving orientifold action. Similar to the case of heterotic string compactification, this condition is merely a constraint on the one particular homology class, and not as constrained as one might thought. There are many solutions of SLAG's, each realizes a particular "splitting" of this homology class. We will comment on this point of view later. 
In the factorized form, the supersymmetry condition is easy to give. Type-IIA string theory on Calabi-Yau three-fold has $D=1 \mathcal{N}=2$ supersymmetry. The orientifold fixed planes preserve one particular half of the bulk supersymmetry, determined by the holomorphic three form. The only way to have $\mathcal{N}=1$ supersymmetry in $D=4$, is that all the D6-branes should preserve the same part of the bulk supersymmetry. As discussed in [43, D6-branes should be related to O6-branes by a SU(3) rotation. In particular, it allows D6 branes to intersect $\mathrm{O} 6$ branes at angles. One of the O6-brane wraps all the $a$-cycle. Then in the factorized form, assume the one cycle in the i-th tori wrapped by the brane intersects the real axis with angle $\theta_{i}$. Then one has the following supersymmetric condition

$$
\theta_{1}+\theta_{2}+\theta_{3}=0
$$

Branes at generic positions provides unitary gauge group and possible chiral fields.

We will be concerned with the deformation of branes and the corresponding change of chiral spectrum. Since we will not consider the fractional branes, the image branes for a brane at generic position under the full orientifold group action will be included in the covering space $T^{6}$, in particular the $\Omega R$ image. The gauge groups arise as follows. $N$ D6branes parallel to orientifold fixed planes produce $\operatorname{USp}(N)$ group, while in generic position with respect to all the orientifold fixed planes, $\mathrm{U}(N)$ group is produced. The orbifold group action reduces it to $\mathrm{U}(\mathrm{N} / 2)$.

The charged chiral multiplets arise from the intersection of the various D6-branes, and carry bifundamental representations of the gauge groups from the intersecting branes. A necessary condition for chiral fermions in $d=4$ is that they carry complex representation of the gauge groups. Therefore it has to involve at least one $\mathrm{U}(N / 2)$ group (there is no possibility of spinor representation of the $\mathrm{SO}(N)$ in this scenario). For $2 N_{a}$ D6-branes intersecting $2 N_{b}$ branes, there are $I_{a b}$ chiral fermions in $\left(N_{a}, \bar{N}_{b}\right)$ from $(a b+b a)$ sector, and $I_{a b^{\prime}}$ chiral fermions from $\left(a b^{\prime}+b^{\prime} a\right)$ sector, where b' is the $\Omega R$ image of $\mathrm{b}$. The intersection number $I_{a b}$ is defined as

$$
I_{a b}=\left[\Pi_{a}\right] \cdot\left[\Pi_{b}\right]=\Pi_{i=3}^{3}\left(n_{a}^{i} m_{b}^{i}-m_{a}^{i} n_{b}^{i}\right) .
$$

Apart from above fields, there are three $\mathcal{N}=1$ chiral multiplets in the adjoint representation associate with each stack of D6-branes, result from spontaneous breaking of $\mathcal{N}=2$ supersymmetry. Chiral multiplet in the antisymmetric representation of $\mathrm{U}(N)$ also arises from $2 N$ D6 branes and its $\Omega R$ image brane. These will parameterize the brane recombination process, which is important for chirality change process we will propose in the following section.

\subsection{Smooth brane deformation and supersymmetric chirality change}

Let us look more carefully at the RR tadpole cancellation constraint eq. (1.4). Expanded out explicitly in the homology basis, it takes the following form

$$
\left[a_{1}\right] \times\left[a_{2}\right] \times\left[a_{3}\right]: \quad \sum_{a} N_{a} n_{a}^{1} n_{a}^{2} n_{a}^{3}=16,
$$




\begin{tabular}{|c|c||c|c||c|c||c|c|}
\hline \multicolumn{2}{|c||}{ Brane type } & \multicolumn{2}{c||}{ Phase I } & \multicolumn{2}{c||}{ Phase II } & \multicolumn{2}{c|}{ Phase III } \\
\hline a & $\Pi_{i}\left(n_{a}^{i}, m_{a}^{i}\right)$ & $N_{a}$ & gauge group & $N_{a}$ & gauge group & $N_{a}$ & gauge group \\
\hline \hline $\mathrm{A}$ & $(1,0) \times(1,1) \times(1,-1)$ & 4 & $\mathrm{U}(2)$ & 4 & $\mathrm{U}(2)$ & 4 & $\mathrm{U}(2)$ \\
\hline $\mathrm{B}$ & $(1,1) \times(1,-1) \times(1,0)$ & 12 & $\mathrm{U}(6)$ & 0 & & 0 & \\
\hline $\mathrm{C}$ & $(1,1) \times(1,0) \times(1,-1)$ & 0 & & 0 & & 12 & $\mathrm{U}(6)$ \\
\hline $\mathrm{D}$ & $(1,0) \times(1,0) \times(1,0)$ & 0 & & 12 & $\mathrm{USp}(12)$ & 0 & \\
\hline $\mathrm{E}$ & $(1,0) \times(0,1) \times(0,-1)$ & 12 & $\mathrm{USp}(12)$ & 12 & $\mathrm{USp}(12)$ & 12 & $\mathrm{USp}(12)$ \\
\hline $\mathrm{F}$ & $(0,1) \times(1,0) \times(0,-1)$ & 16 & $\mathrm{USp}(16)$ & 16 & $\mathrm{USp}(16)$ & 4 & $\mathrm{USp}(4)$ \\
\hline $\mathrm{G}$ & $(0,1) \times(0,-1) \times(1,0)$ & 4 & $\mathrm{USp}(4)$ & $12+4$ & $\mathrm{USp}(16)$ & 16 & $\mathrm{USp}(16)$ \\
\hline
\end{tabular}

Table 2: D6-brane configurations on covering space $T^{6}$ and corresponding gauge groups. I and III have isomorphic gauge groups but different chiral multiplets. They are proposed to connect in a supersymmetric way, via configuration II.

$$
\begin{array}{ll}
{\left[a_{1}\right] \times\left[b_{2}\right] \times\left[b_{3}\right]:} & \sum_{a} N_{a} m_{a}^{1} m_{a}^{2} n_{a}^{3}=-16, \\
{\left[b_{1}\right] \times\left[a_{2}\right] \times\left[b_{3}\right]:} & \sum_{a} N_{a} m_{a}^{1} n_{a}^{2} m_{a}^{3}=-16, \\
{\left[b_{1}\right] \times\left[b_{2}\right] \times\left[a_{3}\right]:} & \sum_{a} N_{a} m_{a}^{1} m_{a}^{2} n_{a}^{3}=-16 .
\end{array}
$$

It is easy to understand why there are four independent equations here. Recall that the orbifold space has $h^{2,1}=3$, add $h^{3,0}=1$, so the homology 3-classes is of complex dimension four. Orientifold action acts as complex conjugation, so its invariant set within the homology classes is of real four dimensional. However, the D6 branes can wrap homology three cycles containing homology classes in the other four dimensional space. Since they are odd under complex conjugation, when the brane and its $\Omega R$ image add together, the components along these direction simply cancel out. This is one hint why the constraint (4.4) is not very restrictive: it is constraint on homology classes, not on actual 3-cycles.

Now it is a simple matter to find different solutions to this constraint equation. Many can be found, as in [8]. Each has different gauge groups and matter content. Our theme in this paper is to find classical process of chirality change, so we would like to know first if there are solutions with isomorphic low energy gauge groups, but different chiral fermions. Indeed, there are examples within this setup, which is listed in table \&.

Three brane configurations are given in the table, which are labelled as I, II and III, together with the gauge groups. Altogether there are seven types of branes (or rather 3 -cycles) involved, listed as type $A, \ldots, G$. The first three types of 3-cycles do not coincide with any orientifold planes and so produce unitary groups, while the last four types coincide with the orientifold plane and produces symplectic gauge group.

Notice I and III have isomorphic low energy gauge groups, which is $\mathrm{U}(2) \times \mathrm{U}(6) \times$ $\mathrm{USp}(12) \times \mathrm{USp}(16) \times \mathrm{USp}(4)$. Now it is clear, that these D6-branes wrap different 3-cycles in the internal Calabi-Yau space, so indeed the gauge groups are different but merely isomorphic. In particular, the chiral spectrum are different. The intersection number of 
the various branes can be easily worked out from (4.6),

$$
\begin{array}{lllll}
I_{A B}=-2, & I_{A D}=0, & I_{A E}=0, & I_{A F}=1, & I_{A G}=-1 ; \\
I_{B D}=0, & I_{B E}=1, & I_{B F}=-1, & I_{B G}=0 ; & \\
I_{A C}=0, & I_{C D}=0, & I_{C E}=1, & I_{C F}=0, & I_{C G}=-1 .
\end{array}
$$

Then the the bifundamental chiral multiplets from the D6-branes intersecting points in phase I and III are

$$
\begin{aligned}
& \mathrm{U}(2) \times \mathrm{U}(6) \times \mathrm{USp}(12) \times \mathrm{USp}(16) \times \mathrm{USp}(4) \\
I: & -2(2, \overline{6}, 1,1,1)+(2,1,1,16,1)-(2,1,1,1,4)+(1,6,12,1,1)-(1,6,1,16,1) . \\
I I I: \quad & -(2,1,1,16,1)+(2,1,1,1,4)+(1,6,12,1,1)-(1,6,1,16,1) .
\end{aligned}
$$

The minus sign denotes the open string with opposite direction, therefore the corresponding chiral multiplets carry complex conjugate representations with respect to the one with positive sign. For example, $-2(2, \overline{6}, 1,1,1)$ is equivalent to 2 chiral multiplets carrying bifundamental representation $(\overline{2}, 6)$ under $\mathrm{U}(2) \times \mathrm{U}(6)$, and being singlet under all the other gauge groups. Again, we have omitted the non-chiral spectrum arising from open string between brane and its $\Omega R$ image, and between a brane with itself.

Evidently, there is a nontrivial change of chirality between I and III. The two chiral multiplets carrying bifundamental representation of $\mathrm{U}(2) \times \mathrm{U}(6)$ in I disappear from III, while there is more subtle change of representation from $(2,16)$ under $\mathrm{U}(2) \times \mathrm{USp}(16)$ in I to $(\overline{2}, 16)$ in III, and from $(2,4)$ under $\mathrm{U}(2) \times \mathrm{USp}(16)$ in I to $(\overline{2}, 4)$ in III. If we ignore the spectator branes in these two configurations, the change can be described in terms of brane types as

$$
12 B+12 B^{\prime}+24 F \rightarrow 12 C+12 C^{\prime}+24 G .
$$

The $\mathrm{F}$ and $\mathrm{G}$ branes have double number than those listed in the table, because their $\Omega R$ image branes are themselves, which are also included in the equation.

One would like to know how configurations I and III are connected. We propose that they can be connected through configuration II. The gauge groups and chiral spectrum of II are as follows,

$$
\begin{aligned}
I I: & \mathrm{U}(2) \times \mathrm{USp}(12) \times \mathrm{USp}(12) \times \mathrm{USp}(16) \times \mathrm{USp}(16) \\
& (2,1,1,16,1)-(2,1,1,1,16) .
\end{aligned}
$$

One realization of the chirality change (4.11) proceeds in two steps as follows. First, from I to II

$$
12 B+12 B^{\prime} \rightarrow 24 D+24 G .
$$

while the second step is from II to III

$$
24 D+24 F \rightarrow 12 C+12 C^{\prime} .
$$

Each only involves the brane configuration in a $T^{4}$, and all the branes involved in one process wrap the common one-cycle in the other torus. Via the above brane "chemical reaction," the chiral spectrum is changed. 


\section{Brane supersymmetric deformation}

Having narrowed down the deformation to a more specific process, one wonders whether this can be realized in a supersymmetric way. At first, the supersymmetric condition (1.5) on the angles of the brane seems very constrained. For example, in (4.13), $\theta_{3}=0$ is fixed, which leaves only one deformation parameter, say $\theta_{1}$, from solving the constraint. On the first $T^{2}$, because of the periodicity of the torus, $\theta_{1}$ can only take discrete values, $\theta=$ $\tan ^{-1}(m / n)$. This might suggest that the supersymmetric solutions are isolated "islands" in the configuration space, and no supersymmetric deformation can connect them.

However it is simple to see that at least some supersymmetric deformations are possible. The McLean theorem [44] states that for a compact special lagrangian submanifold $N$ in a Calabi-Yau manifold, the moduli space of special lagrangian deformation of $N$ is a smooth manifold of dimension $b^{1}(N)$, where $b^{1}(N)$ is the first Betti number of $N$. A heuristic argument for this uses supersymmetry and world-volume gauge theory. Assume a D-brane wraps $N$, which has a $\mathrm{U}(1)$ bundle on it. The flat Wilson lines then parameterize the moduli space of dimension $b^{1}(N)$. Paired with another moduli from minimal area of the string worldsheet in the Calabi-Yau with boundary on the SLAG wrapping the 1-cycle, they form a complex parameter.

Now the orbifold $T^{6} / \mathbb{Z}_{2} \times \mathbb{Z}_{2}$ is simply connected. This is clearly the case as all the one-cycles in the original $T^{6}$ are odd under a certain orbifold group element, and so all disappear after the orbifold projection. However all the special lagrangian 3-cycle $C$ discussed so far has the topology of the tori of real dimension three, and invariant under the whole orbifold group. This means that in the orbifold such a SLAG has moduli space of complex dimension three. A single wrapped D-brane must also have moduli space of complex dimension three. If multiple branes wrap the same SLAG submanifold, the moduli space will increase correspondingly, as the Wilson lines can take value in $\mathrm{U}(n)$ gauge group now.

The consideration of multiple wrapped branes brings us to another important point. In the case at hand, one encounters not merely a single brane wrapping a SLAG, as one notices that there are large number of branes wrapping the same SLAG cycle in the solutions to the constraint equation given above. In addition, a brane's $\Omega R$ image is always present because of the orientifold action. Thus the object to deform, is not certain singly wrapped SLAG submanifold, but the combined 3-cycle with different components wrapped by multiple branes. In particular, splitting and recombining are possible in this setup.

Let us now describe the supersymmetric deformation from I to II (4.12). First observe that the branes involved (B, D and $\mathrm{G}$ in our notation) all wrap the same $a$-cycle in the third torus, i.e. $\theta_{3}=0$, and thus remains the same though the deformation. So the deformation actually happens in four-dimensional complex space $\mathcal{T} \times \mathcal{T}$, and original special lagrangian 3 -cycles reduce to SLAG 2-cycles. The advantage is that algebraic geometry technique can be applied, since SLAG in $4 \mathrm{~d}$ space is just complex curve with respect to a different complex structure, as shown in [43, 45, where the coordinate system is

$$
w_{1}=x_{1}+i x_{2}, \quad w_{2}=y_{1}-i y_{2} .
$$



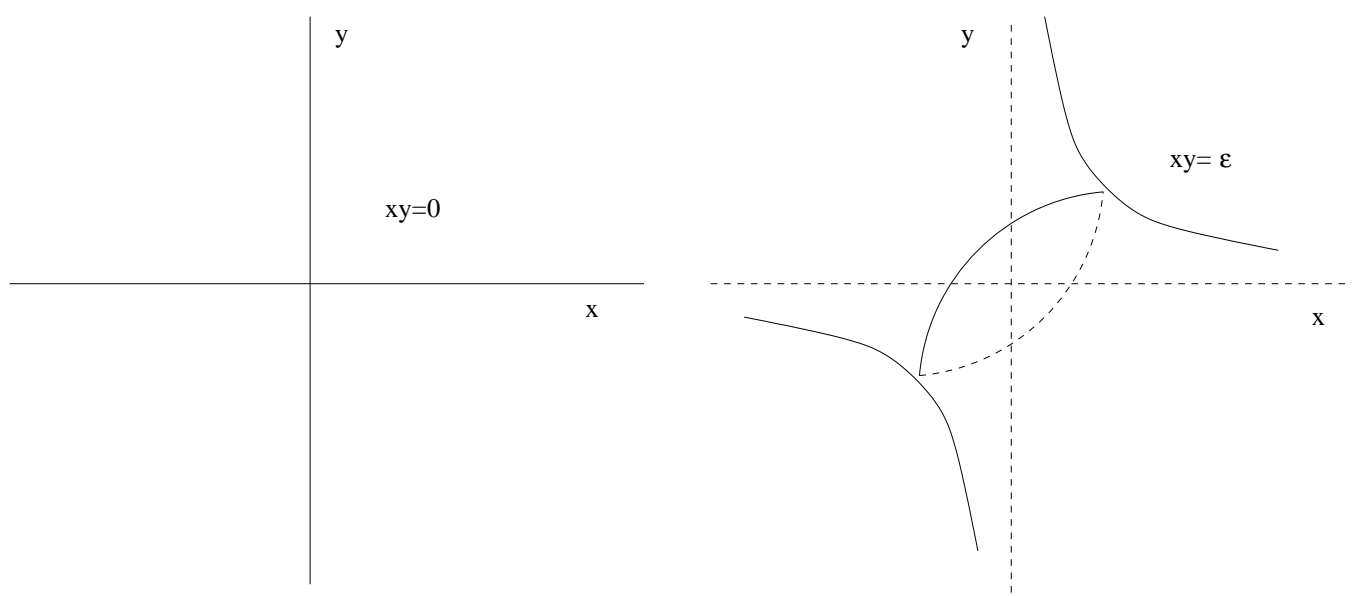

Figure 2: Two intersecting complex curves described by the equation $x y=0$ deform to a a configuration described by the equation $x y=\epsilon . \mathrm{x}$ and $\mathrm{y}$ are complex coordinates, so these are Riemann surfaces, and the one in the second picture is connected. It would be easier to visulize if one regards this as degree- 2 curve defined in a coordinate patch specified by $z \neq 0$ in $P^{2}$ with homogeneous coordinates $(\mathrm{x}, \mathrm{y}, \mathrm{z})$.

Now it is easy to identify B, B', D and G type branes with complex curves in the new coordinate system, as

$$
w_{1}= \pm w_{2} \quad\left(B, B^{\prime}\right) ; \quad w_{2}=0 \quad(D) ; \quad w_{1}=0 \quad(G) .
$$

The orientifold action $\Omega R$ acts as $\left(w_{1}, w_{2}\right) \rightarrow\left(w_{1},-w_{2}\right)$ in the new coordinate system.

Having realized the non-trivial part of the brane configuration as complex curves, the deformation is straightforward. The local picture is illustrated by figure 2 [46]. This picture of brane recombination, while preserving supersymmetry at every stage, is analyzed in detail in [47] for the noncompact target space, which can be viewed as the local model for the process we describe here.

To get a global description, we realize the complex curve as complex intersection of the 4-tori $T^{4}$ with a hypersurface in the product of the projective space $P^{2} \times P^{2}$, deforming the hypersurface will results in a family of holomorphic curves that connects the two configurations. Each $T^{2}$ is nonsingular, can be embedded as cubic curve in $P^{2}$, which in terms of the homogeneous coordinates $\left(x_{i}, y_{i}, z_{i}\right)$ on $P^{2}$ is described by

$$
y_{i}^{2} z_{i}=4 x_{i}^{3}-g_{2} x_{i} z_{i}^{2}-g_{3} z_{i}^{3}, \quad i=1,2 .
$$

These equations define separately a bidegree $(3,0)$ and a $(0,3)$ divisor in $P^{2} \times P^{2}$, which are topologically $T^{2} \times P^{2}$ and $P^{2} \times T^{2}$. Their intersection will produce $T^{2} \times T^{2}$. Notice that the two tori has the same complex structure, so $g_{2}$ and $g_{3}$ are the same for the two factors.

The supersymmetric $B, B^{\prime}, D, G$ branes are complex curves in $T^{4}$. We now find appropriate divisors in $P^{2} \times P^{2}$, so that their intersection with the two divisors 4.16 defining the two embedded tori will give these curves. For this purpose, we need the map from the 
tori to the homogeneous coordinates, which is through Weierstrass $\wp$-function,

$$
\begin{aligned}
& x_{i}=\wp\left(w_{i}\right)=\frac{1}{w_{i}^{2}}+\sum_{0 \neq \lambda \in \Lambda}\left[\frac{1}{\left(w_{i}-\lambda\right)^{2}}-\frac{1}{\lambda^{2}}\right], \\
& y_{i}=\wp^{\prime}\left(w_{i}\right)=\sum_{\lambda \in \Lambda} \frac{-2}{\left(w_{i}-\lambda\right)^{3}}, \quad i=1,2,
\end{aligned}
$$

where the lattice $\Lambda=\{m+n \tau \mid m, n \in \mathbb{Z}\}$ has period determined by the complex structure $\tau$ of the tori, which is $\tau=i$ for the case at hand.

For the $\mathrm{D}$ and $\mathrm{G}$ branes, $w_{2}=0$ and $w_{1}=0$ are mapped to $x_{1}=y_{1}=\infty$ and $x_{2}=y_{2}=\infty$ respectively from Weierstrass function mapping in the coordinate patch $z=1$. Thus they can be identified with equation $z_{2}=0$ and $z_{1}=0$ respectively. There are complications from the degree counting in determining the actual number of wrapped D-branes. An elliptic curve has degree-3 in $P^{2}$, which intersects degree- 1 divisor $z_{1}=0$ at three points. Further intersecting with the torus in the second $P^{2}$, it produces 3 copies of the second torus, which is equivalent to three copies of G-type branes. Similarly, $z_{2}=0$ corresponds to three copies of D-type branes. This is the common feature of embedding tori by Weierstrass form: its intersection with any divisor will be multiple of three. We have arranged our brane solution meet this requirement. Excluding the common factor of 4 for the possible orbifold images, the brane combination $24 D+24 G$ is described by intersection of the tori with the divisor

$$
z_{1}^{2} z_{2}^{2}=0
$$

The $B, B^{\prime}$ branes equations are $w_{1} \pm w_{2}=0$. Now under mapping by Weierstrass function, it leads to $x_{1}=x_{2}$ and $y_{1} \pm y_{2}=0$ in $P^{2} \times P^{2}$. Account for the degree, $3 B+3 B^{\prime}$ in the coordinate patch $z_{1}=z_{2}=1$ is $y_{1}^{2}-y_{2}^{2}=0$. In homogeneous coordinates, this leads to equation

$$
y_{1}^{2} z_{2}^{2}-y_{2}^{2} z_{1}^{2}=0
$$

Because $\Omega R$ acts as $y_{2} \rightarrow-y_{2}$, so above equation is invariant under $\Omega R$, as it should.

Both (4.19) and (4.20) are degree $(2,2)$ divisors on $P^{2} \times P^{2}$. Orientifold action $\Omega R$ acts on the second torus only, $\Omega R: w_{2} \rightarrow-w_{2}$, which can be lifted to $P^{2}$ as $\Omega R: y_{2} \rightarrow-y_{2}$. So if the defining polynomial is even function of $y_{2}$, it is automatically $\Omega R$ invariant. Thus the family of hypersurface which both (4.19) and (4.20) belong to can be written as

$$
F_{(2,2)}\left(x_{1}, y_{1}, z_{1}, x_{2}, y_{2}^{2}, z_{2}\right)=0 .
$$

Deformation within this family can make a transition from configuration I to II.

The intersection of (4.19) and (4.20) with $T^{2} \times T^{2}$ each has the topology of two torus intersecting at one point However the generic member of the family of the $(2,2)$ divisor intersecting with $T^{2} \times T^{2}$ has quite different topology. In the notation of [26], this is specified by a configuration matrix

$$
\left(\begin{array}{l}
2 \\
2
\end{array}|| \begin{array}{lll}
3 & 0 & 2 \\
0 & 3 & 2
\end{array}\right),
$$


where the first column specifies the dimensions of the product projective spaces, and the other columns specify the degrees of the divisors corresponding to each projective space. The configuration represents a family of complete intersections (can be smooth or singular) defined by three polynomial constraints specified by the configuration matrix. It is parameterized by the space of coefficients of the polynomials. A generic member of this family is a smooth complex curve. Let us calculate the genus of this curve $C$.

Let the Kähler forms of the two $P^{2}$ be $J_{1}$ and $J_{2}$. The tangent bundle of $C$ is a subbundle of $T X, X \equiv P^{2} \times P^{2}$, whose quotient is the vector bundle $E$ determined by the polynomials. In other words, there is an exact sequence

$$
0 \rightarrow T C \rightarrow T X|C \rightarrow E|_{C} \rightarrow 0
$$

This implies the relation of the total Chern class

$$
c(C)=\frac{c(X)}{c(E)}=\frac{\left(1+J_{1}\right)^{3}\left(1+J_{2}\right)^{3}}{\left(1+3 J_{1}\right)\left(1+3 J_{2}\right)\left(1+2 J_{1}+2 J_{2}\right)}=-2\left(J_{1}+J_{2}\right) .
$$

The Euler character is obtained from the coefficient of the volume form $J_{1}^{2} J_{2}^{2}$ of $P^{2} \times P^{2}$, in the product

$$
c_{1}(C) \wedge\left(\left(1+3 J_{1}\right)\left(1+3 J_{2}\right)\left(1+2 J_{1}+2 J_{2}\right)\right),
$$

which gives $\chi(C)=-72$. By the familiar Riemann-Hurwitz formula for curves $\chi(C)=$ $2-2 g$, this leads to genus of $C$ being 37 .

Now it is clear that the two configurations that we tried to connect holomorphically are really two degenerate limits of the same family of curves with genus $g=37$. These degenerate configurations develop intersecting points which are singular. But these singular limits have a perfect classical interpretation in the gauge theory, that is the brane splits into multiple branes wrapping different supersymmetric cycles, together with intersections where chiral multiplets live.

As a last step of the proof of the supersymmetric transition realizing chirality change, one notice that the transition from II to III (4.13) can be similarly proved. Switching the role of the second torus with the third torus, it becomes the same process as the transition from I to II.

Thus, we have an explicit example, in which by recombination and splitting, chirality change with isomorphic gauge groups takes place entirely in the moduli space of supersymmetric string vacua.

\section{Conclusions}

We showed that string theory compactifications leading to low energy effective field theories with different chiral matter content are connected even at the classical level, in a way which can be understood from several viewpoints: effective field theory, higher dimensional gauge theory and brane constructions.

The loophole in the naive argument that this is not possible is that the true effective field theory description of the situation is in terms of an infinite gauge group, which in the gauge theory examples is the group of all maps from the internal space to the (finite 
dimensional) gauge group, which contains many copies of any given low energy gauge group. Chirality change takes place by breaking one copy of the low energy gauge group, and restoring a different copy with different chiral matter.

We even found an example in which the transition takes place entirely on the supersymmetric moduli space, in IIa theory with D6-branes. Again, there is no particular paradox in this. In a partially understood sense, this is mirror to similar transitions between type-I vacua (via T-duality/mirror symmetry) and the heterotic string (via S-duality). On the other hand, detailed understanding of the mirror map usually involves $\alpha^{\prime}$ corrections, leaving open the possibility that these corrections are required to get transitions purely through supersymmetric classical vacua. It would be nice to find an example of a transition purely in ten dimensional Yang-Mills theory (we suspect it is possible).

The larger question which this investigation set out to address is to what extent we can think of "all" or large sets of string theory compactifications as described by a single effective field theory, the "effective field theory of everything," with all quantum effects already summarized in the effective lagrangian. Such an effective field theory would have to contain an infinite number of fields, almost all of which are massive in any small region of configuration space, and would probably not be the best way to study any one compactification. Nevertheless it might be very useful as a unifying and organizing concept behind the general discussion.

One can try to propose specific formal realizations of this idea such as the explicit Kaluza-Klein expansion of higher dimensional gauge theory keeping all modes which can become massless anywhere in the region of configuration space of interest, a similar discussion based on world sheet considerations along the lines proposed for toroidal compactification of the heterotic string by Giveon, Porrati and Rabinovici [9], a similar proposal based on string field theory, or otherwise.

However, the main question which will determine whether there is an effective field theory of everything or not, is not a formal question. Rather, it is the physical question of what type of phase transitions connect the different vacua of string/M theory. Certain phase transitions can be easily described in effective field theory language, such as the Coulomb-Higgs transition. Others can be so described by taking advantage of duality, such as the Coulomb-confinement transition. While going beyond the traditional idea of effective field theory, a collection of effective field theories related by explicit dualities, with different theories appropriate in different regions of configuration space, would be a pretty concrete description of the situation.

While such a picture is often cited as the basic picture behind string/M theory duality, it is not yet clear whether one can use it in this literal form, as there is no guarantee that all of the phase transitions one needs to connect the different vacua are of this form. For example, there is no clear argument in supergravity that first order transitions are impossible (indeed one cannot take for granted standard considerations of minimizing the energy).

Another example is a phase transition associated with a non-trivial fixed point theory. While one should certainly not try to describe the physics of the phase transition itself by effective field theory, one might hope that there exists an effective field theory which 
describes all massive deformations of the fixed point theory and the configuration space in its vicinity. This is problematic in the case of a non-trivial fixed point theory which arises at the meeting point of several branches of moduli space, which individually are described by very different sets of degrees of freedom. The simplest effective field theory description would have to contain all of these degrees of freedom, and on each branch give mass to all fields associated with other branches. This is impossible if some of these theories are chiral. One might gain more possibilities by postulating dualities between some of these fields and others, but in any case there are many examples for which such a description is not known at present, and might be impossible.

Our present discussion suggests that, even in cases where this is impossible, it might still be possible to describe all of the branches within a single effective field theory, which connects them up along a different line of deformations. It would not be manifest that they are also connected through non-trivial phase transitions, but this information might be taken into account separately.

One approach to show that this is impossible, would be to find an order parameter which could not change in effective field theory. In the case at hand, we showed that the number of generations is not the relevant order parameter. On the other hand, there is a candidate order parameter in the heterotic string construction. It is $c_{2}(V)$ or equivalently the number of heterotic fivebranes. Thus, to judge the connectedness of this moduli space in effective field theory, we must ask, is $c_{2}(V)$ preserved under all processes which do not pass through non-trivial fixed point theories? While true in the heterotic string, it is not at all obvious in the full string/M theory, again because there are dual descriptions, such as $G_{2}$ compactification, in which it is not obvious how to define this number.

As a final comment, all of the connections we have been talking about between solutions of string theory are visible at low energy. A possibly more important question is whether there are simpler ways to connect solutions in a regime of large quantum and thermal fluctuations, such as one expects to describe early cosmology. Though there is interesting work on the related question of time dependent interpolating solutions (e.g. describing brane-antibrane annihilation), it seems to us that at present nothing is known about this last topic. Perhaps the concrete pictures we have explored in this work could inspire some good questions here.

\section{Acknowledgments}

We thank B. Acharya, F. Bogomolov, A. Langer, N. Seiberg and R. Thomas for valuable discussions.

M.R.D. is supported by a Gordon Moore Visiting Professorship at Caltech, and by DOE grant DE-FG02-96ER40959. C-G.Zhou is supported by DOE grant DE-FG01-00ER45832.

\section{A. Vector bundles $V_{(1,1,1,1,2,2 ; 4,4)}$ on quintic Calabi-Yau}

In this appendix, we prove the proposition in section 3.2 concerning properties of the bundle $V_{(1,1,1,1,2,2 ; 4,4)}$ on quintic Calabi-Yau. 
First, as demonstrated in section 3.2, this bundle has Chern classes

$$
r=4, \quad c_{1}=0, \quad c_{2}=50, \quad c_{3}=90 .
$$

Obvious it is not in the complex moduli space of $T M \oplus \mathcal{O}$. The complex moduli space is parameterized by the map from $\mathcal{O}(1)^{\oplus 4} \oplus \mathcal{O}(2)^{\oplus 2}$ to $\mathcal{O}(4)^{\oplus 2}$. Explicitly,

$$
F=\left(\begin{array}{llll}
f_{1} & f_{2} & \cdots & f_{6} \\
g_{1} & g_{2} & \cdots & g_{6}
\end{array}\right),
$$

where $f_{1}, \cdots f_{4}$ and $f_{1}, \cdots f_{4}$ are homogeneous degree 3 polynomials in homogeneous coordinates $X_{1}, X_{2}, \ldots, X_{5}$ of $P^{4}$, and $f_{5}, f_{6}, g_{5}, g_{6}$ are homogeneous degree 2 polynomials.

We first discuss the question whether generically $\mathrm{V}$ is a vector bundle. This is important for interpretation of this vector bundle as a perturbative heterotic string vacuum. The reason is that singular fibers corresponds to low dimensional branes as small instanton limit of the vector bundle, which signals the existence of the non-perturbative degrees of freedom.

To be rid of singular fibers, the rank of the fibers of $\mathrm{V}$ must be constant over all of the Calabi-Yau space. Because $\mathrm{V}$ is defined as the kernel of the map $F$, this is equivalent to the image of $F$ should have constant rank 2 at each point of Calabi-Yau. Actually for $V_{(1,1,1,1,2,2 ; 4,4)}$ on quintic, we have a stronger result, namely that $\mathrm{V}$ is generically a vector bundle on the whole $P^{4}$, not merely on the quintic hypersurface.

The proof goes as follows. Assume at point $x_{0} \in P^{4}, \operatorname{dim}(\operatorname{Im}(F))<2$. This means that the six two-dimensional vectors $\left.\left(\begin{array}{l}f_{i} \\ g_{i}\end{array}\right)\right|_{x_{0}}, i=1,2, \ldots, 6$ are proportional to each other. This is true if and only if the five functions $\operatorname{det}\left(\begin{array}{ll}f_{1} & f_{i} \\ g_{1} & g_{i}\end{array}\right), i=2, \cdots, 6$ are all equal to zero at $x_{0}$. So the position of singular fibers are the solutions to the 5 equations $f_{1} g_{i}-g_{1} f_{i}=0$, $i=2,3, \ldots, 6$. Generically, there is no solution to these equations as five divisors have no intersection on $P^{4}$ generically. Thus we can always find the map $F$ such that $\mathrm{V}$ is a vector bundle.

Next we discuss the stability property of $V_{(1,1,1,1,2,2 ; 4,4)}$. We would like to know whether there exists a stable vector bundle in the same complex deformation class of $\mathrm{V}$. This is again by analysis of F. We can only obtain partial results, namely, we can prove that if choosing appropriately, $\mathrm{V}$ is stable for a class of subsheaves which also has monad like description. In general the stability property of a vector bundle is a very hard problem practically, especially for high rank vector bundles at high dimensional space. In view of this difficulty, although we only can prove the stability partially, we feel that it is a strong evidence that $\mathrm{V}$ can be a $\mathrm{N}=1$ perturbative heterotic string vacuum.

A vector bundle is stable if all its subsheaves $V^{\prime}$ with strictly smaller rank satisfies the condition $\frac{c_{1}\left(V^{\prime}\right)}{\operatorname{rank}\left(V^{\prime}\right)}<\frac{c_{1}(V)}{\operatorname{rank}(V)}$. Here $\mu(V)=c_{1}(V) / \operatorname{rank}(V)$ is called the slope of the vector bundle, and the stability criterion we use here is called slope stability condition. This is the appropriate stability condition at the large volume limit of the Calabi-Yau space, otherwise we should use the П-stability suitable for stringy Calabi-Yau.

Any vector bundle $V^{\prime}$ has injective resolution, which is a complex

$$
0 \rightarrow V^{\prime} \rightarrow W_{1} \stackrel{G}{\rightarrow} W_{2} \rightarrow \cdots
$$


whose cohomology is trivial except at the first term. The vector bundles $W_{i}$ are injective objects in the derived category of torsionless sheaves. In case of $P^{4}$, these injective objects are simply direct sum of line bundles of various degrees, $W_{i}=\oplus_{k} \mathcal{O}(k)$. For $V^{\prime}$ to be subsheaf of $\mathrm{V}$ means the following diagram commutes,

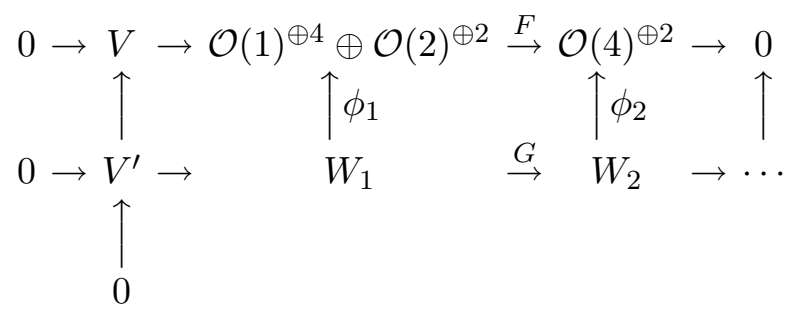

On $P^{4}$, a generic sheaf has an injective resolution with five terms, by analogous construction of Koszul resolution. Because of difficulty to list all of these sheaves, we will merely check those subsheaves of $\mathrm{V}$ such that they can be similar described as a kernel of a surjective map between two direct sum of line bundles. This is the same as saying V' has the same description as $\mathrm{V}$.

The injective resolution of a sheaf is not unique. We assume a minimal resolution of V'. This means that we choose $W_{i}$ 's to have minimal rank among all the equivalent injective resolutions of $\mathrm{V}^{\prime}$. This condition, combined with the injection of map $0 \rightarrow V^{\prime} \rightarrow V$, yields the injection $W_{1} \rightarrow \mathcal{O}(1)^{\oplus 4} \oplus \mathcal{O}(2)^{\oplus 2}$. But $\phi_{2}$ may has a nontrivial kernel, and yet the diagram still commutes. This causes possible complications.

The potentially destabilizing subsheaf $V^{\prime}$ satisfies the following constraints:

1. $C_{1}\left(V^{\prime}\right)=c_{1}\left(W_{1}\right)-c_{1}\left(W_{2}\right)>0$;

2. $W_{1} \stackrel{\phi_{1}}{\rightarrow} \mathcal{O}(1)^{\oplus 4} \oplus \mathcal{O}(2)^{\oplus 2}$ is injective;

3. $G$ and $\phi_{i}$ should all have nonnegative degrees.

There are only a finite number of possibilities. Especially condition (2) restricts the line bundles in $W_{1}$ to have degree $2,1,0, \ldots$.

Case I. $\mathcal{O}(2) \subset W_{1}$, and this line bundle is mapped to $W_{2}$ nontrivially. There are three possibilities. (The factor $\mathcal{O}(2)$ in $W_{2}$ does not give anything nontrivial. $\mathcal{O}(1)$ in $W_{2}$ is not involved.)

1. $W_{2}=\mathcal{O}(3) . W_{1}$ must be equal to one of the $\mathcal{O}(2)$ factors (assume to be the last factor) after a suitable linear transformation. Thus we have commuting diagram

$$
\begin{aligned}
& \mathcal{O}(2) \stackrel{F}{\rightarrow} \mathcal{O}(4)^{\oplus 2} \rightarrow 0 \\
& \uparrow \phi_{1} \quad \uparrow \phi_{2} \\
& \mathcal{O}(2) \stackrel{G}{\rightarrow} \mathcal{O}(3) \quad \rightarrow 0
\end{aligned}
$$

The commuting condition, $\phi_{3} \cdot G=F$ is

$$
\left(\begin{array}{l}
\left(\phi_{2}\right)_{1} \\
\left(\phi_{2}\right)_{2}
\end{array}\right) G=\left(\begin{array}{l}
f_{6} \\
g_{6}
\end{array}\right)
$$


where $\left(\phi_{2}\right) i$ and $G$ are homogeneous degree one polynomials, and $f_{6}, g_{6}$ are homogeneous degree two polynomials. This should be regarded as an algebraic equation, i.e. we should expand out both side and compare coefficients of monomials. In general, this results in a set of quadratic equations for the coefficients of $\left(\phi_{2}\right) i$ and $G$, and the coefficients of $f_{6}, g_{6}$ are given. Counting the number of unknowns $(3 \times 5=15)$ and the number of equations (which is the number of monomials in $f_{6}$ and $g_{6}$, i.e. $2 \times 15=30$ ), it is obviously an over-determined set of equations. So generically, there is no solution for $\phi_{2}$ and $G$.

2. $W_{2}=\mathcal{O}(3) \oplus \mathcal{O}(3)$. Simple dimensional counting similar to above case does not work. We have to consider all the conditions. Including the $c_{1}\left(V^{\prime}\right)$ positive condition, this occurs only if the resolution of $V^{\prime}$ is

$$
0 \rightarrow \mathcal{O}(1)^{\oplus 2} \oplus \mathcal{O}(2)^{2} \stackrel{G}{\rightarrow} \mathcal{O}(3)^{\oplus 2} \rightarrow 0 .
$$

The image of $\phi_{1}$ can be made in a five dimensional subspace via a trivial linear transformation, and the only nontrivial part of $\phi_{1}$ is $\mathcal{O}(1)^{3} \rightarrow \mathcal{O} 2^{2}$. Spell out the commuting condition, we have

$$
\left(\begin{array}{ll}
\left(\phi_{2}\right)_{11} & \left(\phi_{2}\right)_{12} \\
\left(\phi_{2}\right)_{21} & \left(\phi_{2}\right)_{22}
\end{array}\right) \cdot\left(\begin{array}{lllll}
G_{11} & G_{12} & G_{13} & G_{14} & G_{15} \\
G_{21} & G_{22} & G_{23} & G_{24} & G_{25}
\end{array}\right)=\left(\begin{array}{lllll}
f_{2} & f_{3} & f_{4} & f_{5} & f_{6} \\
g_{2} & g_{3} & g_{4} & g_{5} & g_{6}
\end{array}\right) \cdot \phi_{1} .
$$

There are 160 unknowns in the coefficients of $\phi_{1}, \phi_{2}$ and $G$, but there are 270 equations. Excluding the linear transformation between the equivalent line bundles in the same sire sum, this reduce the equation to 270. Again, this is an over-determined set of equations, and generically there is no solutions.

3. $W_{2}=0$. This is the same as saying $\mathrm{V}$ contains $\mathcal{O}(2)$ as a sub-bundle. Obviously, a generically $\mathrm{F}$ will involve both $\mathcal{O}(2)$ factors, and there should not be such nontrivial sub-line bundle $\mathrm{n}$ V. Direct proof via dimension counting is very similar to case one, and we will omit the details.

Since we require that the resolution of $V^{\prime}$ is minimal, so if there is a factor $\mathcal{O}(2)$ in $W_{1}$ was involved trivially, there must be a resolution with smaller resolution without this factor. So we can just consider that $W_{1}$ only contains $\mathcal{O}(1)$ factors.

Case II. $W_{1}=\mathcal{O}(1)^{\oplus m}, m=1,2,3,4$. We have the following possibilities.

1. $W_{1}=\mathcal{O}(1), W_{2}=0$. This is similar to case I.3. There are no solutions generically.

2. $W_{1}=\mathcal{O}(1)^{2}$. By a trivial linear transformation of the two line bundles, this decomposes into direct sum of two series. No solutions generically.

3. $W_{1}=\mathcal{O}(1)^{3}$. The only nontrivial case is $W_{2}=\mathcal{O}(2)$. Similar to case I.2. There are 135 unknowns, but there are 270 equations. Generically, there is no solution.

4. $W_{1}=\mathcal{O}(1)^{4}$. The nontrivial cases are $W_{2}=\mathcal{O}(2)$ or $\mathcal{O}(3)$.Similar to case I.2. There are 170 and 110 unknowns respectively,, and 340 equations for both case. Again there is no solutions generically. 
All the other possibilities will involve the cases considered above. We can thus draw the conclusion that vector bundle $V_{(1,1,1,1,2,2 ; 4,4)}$ is stable against a class of subsheaves that has can be similarly described as $V$ itself.

\section{B. On finiteness of number of solutions to monad construction of vector bundles}

In this section, following [27, we prove there are only finite number of solutions to the sets of holomorphic vector bundles which are described by monad construction and satisfy anomaly cancellation condition on a Calabi-Yau manifold described by intersecting hyperplanes in weighted projective space.

The rank-n vector bundle $\mathrm{V}$ is parameterized by two sets of positive integers $\left(n_{i} ; m_{j}\right)$ $(i=1, \ldots, M+n ; j=1, \ldots, M)$ constrained by

$$
\sum_{i=0}^{n+M} n_{i}=\sum_{j=1}^{M} m_{j}, \quad \sum_{i=0}^{n+M} n_{i}^{2}+B=\sum_{j=1}^{M} m_{j}^{2}, \quad m_{j}>n_{i}>0, \quad \forall i, j .
$$

where constant B is defined in (3.11) and fixed by the second Chern class of the Calabi-Yau manifold. We define

$$
S \equiv \sum_{i=0}^{n+M} n_{i}=\sum_{j=1}^{M} m_{j}
$$

for convenience.

We first prove an inequality which will be essential for the proof,

$$
B \geq S \text {. }
$$

The proof is as follows:

$$
\begin{aligned}
B & \equiv \sum_{j=1}^{M} m_{j}^{2}-\sum_{i=0}^{n+M} n_{i}^{2} \\
& \geq\left(n_{\max }+1\right) \cdot \sum_{j=1}^{M} m_{j}-\sum_{i=0}^{n+M} n_{i}^{2} \\
& =S+\sum_{i=0}^{n+M} n_{\max } n_{i}-\sum_{i=0}^{n+M} n_{i}^{2} \\
& \geq S
\end{aligned}
$$

Now we proceed in the following steps:

1. $\mathrm{n}$ is a finite positive integer. The connection on the holomorphic vector bundle takes value in a subgroup $G$ of $E_{8}$, whose rank is finite. Further we consider the vector bundle $V$ being associated with the vector representation of $G$, whose rank n is also finite. 
2. $\mathrm{M}$ is finite, actually, $M \leq \min \left\{\frac{B}{2}, B-n\right\}$. The proof is as follows: from $B \geq S$, one has

$$
B \geq \sum_{j=1}^{M} m_{j} \geq 2 M
$$

as $m_{j}>n_{i}>0$ so $m_{i} \geq 2$. This gives $M \leq \frac{B}{2}$. A similar consideration for $S=\sum_{i=0}^{n+M} n_{i}$ produces the other inequality.

3. The maximal value $m_{\max }$ of $m_{j}$ is finite, $m_{\max } \leq B-2(M-1)$. The proof is as follows,

$$
B \geq S=\sum_{j=1}^{M} m_{j} \geq m_{\max }+2(M-1) .
$$

4. The maximal value of $n_{i}$ is also finite. This is a simple result of constraint $m_{j}>n_{i}>$ 0 .

Thus we see that on a fixed Calabi-Yau manifold with fixed B, all the parameters $\left(n, M, m_{i}, n_{j}\right)$ are constraint by certain maximal values. Thus the number of solutions to the above equations are finite.

\section{References}

[1] P. Candelas, G.T. Horowitz, A. Strominger and E. Witten, Vacuum configurations for superstrings, Nucl. Phys. B 258 (1985) 46.

[2] C.M. Hull and E. Witten, Supersymmetric sigma models and the heterotic string, Phys. Lett. B $160(1985) 398$.

[3] E. Witten, New issues in manifolds of SU(3) holonomy, Nucl. Phys. B 268 (1986) 79.

[4] P. Hořava and E. Witten, Heterotic and type-I string dynamics from eleven dimensions, Nucl. Phys. B 460 (1996) 506 hep-th/9510209; Eleven-dimensional supergravity on a manifold with boundary, Nucl. Phys. B 475 (1996) 94 hep-th/9603142.

[5] S. Kachru and E. Silverstein, Chirality-changing phase transitions in $4 d$ string vacua, Nucl. Phys. B 504 (1997) 272 hep-th/9704185;

E. Silverstein, Closing the generation gap, Nucl. Phys. 68 (Proc. Suppl.) (1998) 274 hep-th/9709209.

[6] B.A. Ovrut, T. Pantev and J. Park, Small instanton transitions in heterotic M-theory, J. High Energy Phys. 05 (2000) 045 hep-th/0001133.

[7] M.B. Green, J.H. Schwarz and E. Witten, Superstring theory. Vol II, loop amplitudes, anomalies and phenomenology, Cambridge University Press, Cambridge 1987.

[8] M. Cvetič, G. Shiu and A.M. Uranga, Chiral four-dimensional $N=1$ supersymmetric type-IIA orientifolds from intersecting d6-branes, Nucl. Phys. B 615 (2001) 3 hep-th/0107166.

[9] A. Giveon, M. Porrati and E. Rabinovici, Target space duality in string theory, Phys. Rept. 244 (1994) 77 hep-th/9401139. 
[10] M. Atiyah, R. Bott, The Yang-Mills equations over Riemann surfaces, Phil. Trans. R. Soc. Lond. A308 (1982) 523-615

[11] A. Salam and J. Strathdee, On Kaluza-Klein theory, Ann. Phys. (NY) 141 (1982) 316.

[12] S. Randjbar-Daemi, A. Salam and J. Strathdee, Spontaneous compactification in six-dimensional Einstein-Maxwell theory, Nucl. Phys. B 214 (1983) 491.

[13] A. Grothendieck, Sur la classification des fibrés holomorphes sur la sphère de Riemann, Am. J. Math. 79 (1957) 121-38.

[14] Z. Guralnik and S. Ramgoolam, From 0-branes to torons, Nucl. Phys. B 521 (1998) 129 hep-th/9708089.

[15] C. Okonek, M. Schneider and H. Spindler, Vector bundles on complex projective spaces, Birkhauser, Boston 1980.

[16] L. Pottier, Lectures on vector bundles, Cambridge University Press, Cambridge 1997.

[17] A. Pressley and G. Segal, Loop groups, Clarendon Press, Oxford 1988.

[18] S.K. Donaldson and P.B. Kronheimer, The geometry of four-manifolds, Clarendon Press, Oxford 1990.

[19] J.-M. Drezet, Exceptional bundles and moduli space of stable sheaves on $P^{n}$, in Vector bundles in algebraic geometry: Durham, 1993, Cambridge University Press, Cambridge 1995.

[20] D-E Diaconesco, G. Moore and E. Witten, E(8) gauge theory, and a derivation of $K$ theory from $M$ theory, Adv. Theor. Math. Phys. 6 (2003) 1031.

[21] M. Maruyama, On boundedness of torsion free sheaves, J. Math. Kyoto Univ. 21 (1981) 673-701.

[22] A. Langer, Semistable sheaves in positive characteristic, math.AG/0312260.

[23] J. Distler and B.R. Greene, Aspects of (2,0) string compactifications, Nucl. Phys. B 304 (1988) 1.

[24] S. Kachru, Some three generation (0,2) Calabi-Yau models, Phys. Lett. B 349 (1995) 76 hep-th/9501131.

[25] P. Candelas, M. Lynker and R. Schimmrigk, Calabi-Yau manifolds in weighted $P^{4}$, Nucl. Phys. B 341 (1990) 383.

[26] T. Hübsch, Calabi-Yau manifolds: A bestiary for physicists, World Scientific, Singapore 1992.

[27] A. Lukas and B.A. Ovrut, Symmetric vacua in heterotic M-theory, hep-th/9908100.

[28] M. Berkooz et al., Anomalies, dualities and topology of $D=6 N=1$ superstring vacua, Nucl. Phys. B 475 (1996) 115 hep-th/9605184.

[29] R. Blumenhagen, L. Görlich and B. Körs, Supersymmetric orientifolds in 6D with D-branes at angles, Nucl. Phys. B 569 (2000) 209 hep-th/9908130.

[30] R. Blumenhagen, L. Görlich and B. Körs, Supersymmetric $4 D$ orientifolds of type-IIA with D6-branes at angles, J. High Energy Phys. 01 (2000) 040 hep-th/9912204.

[31] S. Förste, G. Honecker and R. Schreyer, Supersymmetric $\mathbb{Z}_{N} \times \mathbb{Z}_{M}$ orientifolds in 4 d with D-branes at angles, Nucl. Phys. B 593 (2001) 127 hep-th/0008250]. 
[32] R. Blumenhagen, V. Braun, B. Körs and D. Lüst, Orientifolds of K3 and Calabi-Yau manifolds with intersecting D-branes, J. High Energy Phys. 07 (2002) 026 hep-th/0206038.

[33] R. Blumenhagen, V. Braun, B. Körs and D. Lüst, The standard model on the quintic, hep-th/0210083.

[34] I. Brunner and K. Hori, Orientifolds and mirror symmetry, hep-th/0303135.

[35] S. Govindarajan and J. Majumder, Orientifolds of type-IIA strings on Calabi-Yau manifolds, Pramana 62 (2004) 711-716 [hep-th/0305108.

[36] S. Govindarajan and J. Majumder, Crosscaps in gepner models and type-IIA orientifolds, $\square$. High Energy Phys. 02 (2004) 026 hep-th/0306257.

[37] R. Blumenhagen, Supersymmetric orientifolds of Gepner models, J. High Energy Phys. 11 (2003) 055 hep-th/0310244.

[38] I. Brunner, K. Hori, K. Hosomichi and J. Walcher, Orientifolds of Gepner models, hep-th/0401137.

[39] R. Blumenhagen and T. Weigand, Chiral supersymmetric gepner model orientifolds, J. High Energy Phys. 02 (2004) 041 hep-th/0401148.

[40] M. Cvetič, P. Langacker and G. Shiu, A three-family standard-like orientifold model: Yukawa couplings and hierarchy, Nucl. Phys. B 642 (2002) 139 hep-th/0206115.

[41] M. Cvetič, I. Papadimitriou and G. Shiu, Supersymmetric three family SU(5) grand unified models from type-IIA orientifolds with intersecting D6-branes, Nucl. Phys. B 659 (2003) 193 hep-th/0212177.

[42] C. Vafa and E. Witten, On orbifolds with discrete torsion, J. Geom. Phys. 15 (1995) 189 hep-th/9409188.

[43] M. Berkooz, M.R. Douglas and R.G. Leigh, Branes intersecting at angles, Nucl. Phys. B 480 (1996) 265 hep-th/9606139.

[44] R.C. McLean, Deformation of calibrated submanifolds, Communications in Analysis and Geometry 6 (1998) 705-747.

[45] D. Joyce, Lectures on special lagrangian geometry, math.DG/0111111.

[46] C.G. Callan Jr. and J.M. Maldacena, D-brane approach to black hole quantum mechanics, Nucl. Phys. B 472 (1996) 591 hep-th/9602043.

[47] J. Erdmenger, Z. Guralnik, R. Helling and I. Kirsch, A world-volume perspective on the recombination of intersecting branes, J. High Energy Phys. 04 (2004) 064 hep-th/0309043. 


\section{Erratum}

Reference [22] should be

[22] A. Langer, Semistable sheaves in positive characteristic, Ann. Math. 159 (2004) 251.

The author has explained to us that a complicated but explicit bound on $c_{3}$ can be extracted from these results. 